\title{
EXISTENCE OF CONSERVATION LAWS AND CHARACTERIZATION OF RECURSION OPERATORS FOR COMPLETELY INTEGRABLE SYSTEMS
}

\author{
JOSEPH GRIFONE AND MOHAMAD MEHDI
}

\begin{abstract}
Using the Spencer-Goldschmidt version of the Cartan-Kähler theorem, we give conditions for (local) existence of conservation laws for analytical quasi-linear systems of two independent variables. This result is applied to characterize the recursion operator (in the sense of Magri) of completely integrable systems.
\end{abstract}

\section{INTRODUCTION}

A conservation law for a (1-1) tensor field $h$ on a manifold $M$ is a 1-form $\theta$ which satisfies $d \theta=0$ and $d h^{*} \theta=0$, where $h^{*}$ is the transpose of $h:\left(h^{*} \theta\right)(X):=\theta(h X)$.

Conservation laws arise, for example, in the following classical problem. Consider a system of $n$ quasi-linear equations in two independent variables :

$$
\frac{\partial x^{i}}{\partial u}+h_{j}^{i}(x) \frac{\partial x^{j}}{\partial v}=0 \quad(i=1, \ldots, n)
$$

(repeated indices being summed from 1 to $n$ ). If $\theta:=\lambda_{i}(x) d x^{i}$ is a conservation law with respect to the (1-1) tensor field defined by the matrix $h_{j}^{i}$, there exist locally two functions $f$ and $g$ so that $\theta=d f$ and $h^{*} \theta=d g$, i.e. $\lambda_{i}=\frac{\partial f}{\partial x^{i}}$ and $h_{j}^{i} \lambda_{i}=\frac{\partial g}{\partial x^{j}}$, and we have

$$
0=\lambda_{i} \frac{\partial x^{i}}{\partial u}+\lambda_{i} h_{j}^{i} \frac{\partial x^{j}}{\partial v}=\frac{\partial f}{\partial x^{j}} \frac{\partial x^{j}}{\partial u}+\frac{\partial g}{\partial x^{j}} \frac{\partial x^{j}}{\partial v} .
$$

Then for any solution $x^{i}(u, v)$ of the system $(*)$, we have

$$
\frac{\partial f(x(u, v))}{\partial u}+\frac{\partial g(x(u, v))}{\partial v}=0
$$

and it contains a conservation law in the sense of Lax [9].

Many interesting properties have been developed for systems of partial differential equations which contain conservation laws, and in particular for systems which can be expressed entirely in terms of conservation laws. It is therefore of interest to know when these conditions are satisfied.

More recently, conservation laws have been employed by Magri in his classical paper concerning Hamiltonian completely integrable systems [11]: $h$ is the recursion

Received by the editors November 28, 1994 and, in revised form, April 3, 1996.

1991 Mathematics Subject Classification. Primary 35G20, 35N10; Secondary 58F07, 58G30.

Key words and phrases. Quasi-linear partial differential equations, conservation laws, completely integrable systems, overdetermined system of partial differential equations, Cartan-Kähler theorem, Poisson-Nijenhuis manifolds. 
operator and conservation laws are called by Magri "fundamental forms". They occur in the following manner.

Let $P$ be a Poisson structure on $M$. We can identify $P$ with a map $\bar{P}: T^{*} M \longrightarrow$ $T M$. Let $X \in \mathcal{X}(M)$ be a Hamiltonian field $\left(X=\bar{P} d H\right.$ with $\left.H \in \mathcal{C}^{\infty}(M)\right)$. The system defined by $X$ is called bi-Hamiltonian if there exists another Poisson structure $Q$, "compatible" with $P$ (i.e. $P+Q$ is a Poisson tensor), for which $X$ is Hamiltonian too, i.e. there exists $K \in \mathcal{C}^{\infty}(M)$ so that $X=\bar{Q} d K$. Note that $H$ and $K$ are first integrals of the system, in involution with respect to $P$ and to $Q$. Then the existence of a Hamiltonian field is equivalent to the existence of $H, K \in \mathcal{C}^{\infty}(M)$ for which

$$
\bar{P} d H=\bar{Q} d K .
$$

Suppose that $P$ is invertible (in fact one works on the leaves of the foliation defined by $\operatorname{Im} P$ ). The above equation can be written as $d H=\bar{P}^{-1} \circ \bar{Q} d K$, or

$$
h^{*} d K=d H
$$

where $h=\left(\bar{P}^{-1} \circ \bar{Q}\right)^{*}$. Then $d K$ is a conservation law for $h$.

One can easily prove that the compatibility condition for $P$ and $Q$ is equivalent to the fact that the Nijenhuis bracket $[h, h]$ vanishes, and it is not difficult to show that $h^{*} \theta, h^{*^{2}} \theta, \ldots, h^{*^{i}} \theta, \ldots$ are conservation laws for $h$ if $\theta$ is a conservation law. Then we have (locally) a sequence of first integrals for the system, which, since $[h, h]=0$, are in involution. If these first integrals are functionally independent and their number is equal to the number of degrees of freedom, then the system is completely integrable in the sense of Liouville. It is natural to ask when these conditions for $h$ are satisfied.

Locally, giving a conservation law is equivalent to giving a function $f$ such that

$$
\left(d \circ h^{*} \circ d\right)(f)=0 .
$$

Thus the study of the local existence of conservation laws is equivalent (in an analytic context) to the study of the formal integrability of the differential operator $d \circ h^{*} \circ d$. This problem has already been studied by Osborn, who, using Cartan's theory of exterior differential systems, showed the existence of conservation laws when $h$ has constant coefficients in a suitable coordinate system [13].

For a generalization of this result, as Osborn [14] has already noted, it is useful to decompose $h$ on cyclic subspaces. In a previous paper [2] we proved, using the Spencer-Goldschmidt version of the Cartan-Kähler theorem, that there are no obstructions to solving the problem when $h$ is cyclic.

In the general case further difficulties arise. In fact, the differential operators which appear naturally in the construction of the exact sequence for the first prolongation of the symbol, i.e. $d$ and $d_{h}$, do not suffice to characterize the obstruction space, because the dimension of this space is too big. However, using the fact that the iterations of a conservation law by $h$ are also conservation laws, we are able to determine supplementary obstructions.

The main result of the present paper, whose essential ideas were given in Mehdi's thesis in 1991 [12], can be expressed as follows. Let $h$ be an analytic (1-1) tensor field with $[h, h]=0$, and suppose that its "algebraic type" is constant. For any eigenvalue $\lambda$ of $h$ whose multiplicity is $p$ in the minimal polynomial, we introduce the following notation: $g:=(h-\lambda i d), C_{s} \in \wedge^{2}\left(\operatorname{Ker} g^{s}\right)^{*} \otimes T M($ with $s=(1, \ldots, p))$ 
defined by

$$
C_{s}(X, Y):=-g^{s}[X, Y]+s\left(d \lambda \wedge g^{s-1}\right)(X, Y)
$$

and $C_{s}^{*}: T^{*} M \rightarrow \wedge\left(\operatorname{Ker} g^{s}\right)^{*}$ defined by

$$
C_{s}^{*}(\omega)(U, V):=\omega\left(C_{s}\right)(U, V)
$$

Theorem. 1. A 1-form $\omega_{o} \in T_{x_{o}}^{*} \simeq\left(J_{1}\right)_{x_{o}} \mathbb{R}$ can be lifted to a second order solution of the differential operator $d h^{*} d$ if and only if

$$
C_{s}^{*}\left(\omega_{o}\right)=0
$$

for any eigenvalue and for any $s$

2. The differential operator $d h^{*} d$ is formally integrable at $x_{o}$ if and only if for any eigenvalue and for any $s$

$$
\left(p_{1} C_{s}^{*}\right)\left(F_{o}\right)=0
$$

for every second order solution $F_{o}$, where $p_{1} C_{s}^{*}$ is the first prolongation of $C_{s}^{*}$

Thus in the analytic context the 1 -forms at $x_{o}$ which can be lifted to a germ of conservation laws are just the forms $\omega_{o}$ satisfying $C_{s}^{*}\left(\omega_{o}\right)=0$, if the differential operator is formally integrable.

If we consider the particular case of the existence of a "complete" system of conservation laws, that is, every 1 -form at $x_{o}$ can be extended to a germ of conservation laws (this being the case for "completely integrable systems"), a geometrical interpretation of this result can be given. The obstructions express the integrability of the "characteristic flags" $\mathrm{Ker}^{s}(s=1,2, \ldots, p)$ and the invariance of the eigenvalues on the "maximal leaves" Ker $g^{p}$ or on the "maximal proper leaves" Ker $g^{p-1}$. They can be described by saying that $h$ can be written, in suitable coordinates, with affine coefficients. More precisely, we have:

Corollary. The following two statements are equivalent:

a) There exists a neighborhood $U$ of $x_{0}$ which admits a complete system of germs of conservation laws (i.e. each $\omega_{o} \in T_{x_{o}}^{*}$ can be extended to a germ of conservation laws on $U$ ).

b) Let $\lambda$ be an eigenvalue of $h$ with multiplicity $p$ in the minimal polynomial. If $p=1$, then the characteristic leaves are 1-dimensional; if $p \geq 2$ then either $\lambda$ is constant on the maximal leaves, or $\lambda$ is constant on the maximal proper leaves, which, in this case, are necessarily 1-codimensional in the maximal leaves.

Note. Recently, F. J. Turiel [17], using a completely different technique, obtained a nice generalization of this last result in the $\mathcal{C}^{\infty}$ case (the essential ideas appeared in a different context in his earlier paper [16]). His result agrees with our normal form in Theorem 3.4.

Acknowledgment. We thank J. Gasqui for introducing us to the techniques used in the paper and for pointing the problem out to us. We would also like to thank D. DeTurck, A. Reyman and F.J. Turiel for discussions and encouragement while the work was in progress. 


\section{INVOLUTIVITY OF THE SYMBOL AND CHARACTERIZATION OF THE OBSTRUCTION SPACE}

1.1. Conservation laws. We use the same notations as in [2]. In particular, $M$ being a differentiable manifold, we denote by $T$ and $T^{*}$ the tangent and the cotangent bundles and by $\Lambda^{k} T^{*}$ and $S^{k} T^{*}$ the bundles of the skew-symmetric and symmetric $k$-forms. Let $E \longrightarrow M$ be a vector bundle; $\underline{E}$ will denote the sheaf of germs of the sections of $E$, and $J_{k}(E)$ the vector bundle of the $k$-jets of the sections of $E$. If $L$ is a field of vector valued forms $\left(L \in \wedge T^{*} \otimes T\right)$, then we denote by $i_{L}$ and $d_{L}$ the derivations of type $i_{*}$ and $d_{*}$ associated to $L$ (cf.[5]). We recall here briefly only what is needed in this paper.

If $h$ is a (1-1) tensor field, one defines

$$
\begin{aligned}
i_{h} \omega\left(X_{1}, \ldots, X_{p}\right) & :=\sum_{i=1, \ldots, p} \omega\left(X_{1}, \ldots, h X_{i}, \ldots, X_{p}\right) \quad \text { for } \omega \in \Lambda^{p} T^{*}, \\
d_{h} \omega & :=i_{h} d \omega+(-1)^{p} d i_{h} \omega \quad \text { for } \omega \in \underline{\Lambda}^{p} T^{*} .
\end{aligned}
$$

Also, $[h, h](X, Y):=[h X, h Y]+h^{2}[X, Y]-h[h X, Y]-h[X, h Y][h, h]$ is the so-called "Frölicher-Nijenhuis torsion", which will play a central role in our paper. As is well known, one has $d_{h}^{2}=0$ if and only if $[h, h]=0$

We shall use the Spencer-Goldschmidt version [7] [15] of the Cartan-Kähler theorem [3] [10]. For a complete exposition see [1]; a very accessible presentation is given in [6]. In Appendix 2 we give some basic elements in the linear case in order to explain our notation and to help the reader to follow the demonstration.

Definition 1.1. Let $h \in \underline{T^{*} \otimes T}$; a conservation law for $h$ is a field of 1 -forms $\theta \in \underline{T}^{*}$ which satisfies $d \theta=0$ and $d_{h} \theta=0$.

In this paper we suppose that $h$ has a constant algebraic type. This means that if $P_{h}(X):=\left(X-\lambda_{1}\right)^{\alpha_{1}} \ldots\left(X-\lambda_{p}\right)^{\alpha_{p}}$ is the characteristic polynomial of $h$, then the dimensions of the spaces $\operatorname{Ker}\left(h-\lambda_{i}\right)_{i}^{r}$ (for $i=1, \ldots, p$ and $r_{i}=1, \ldots, \alpha_{i}$ ) and the $\alpha_{i}$ are locally constant.

Locally, to give a conservation law is equivalent to giving a function $f$ such that

$$
d d_{h} f=0
$$

The following proposition is crucial for the rest of the paper:

Proposition 1.1. Let $h \in \underline{T^{*} \otimes T}$ satisfy $[h, h]=0$, and let $\rho_{i}: \wedge^{2} T^{*} \longrightarrow$ $\wedge^{2} T^{*}(i \geq 1)$ be the maps defined by $\left(\rho_{i} \omega\right)(X, Y):=\sum_{k=1}^{i} \omega\left(h^{i-k} X, h^{k-1} Y\right)$. Then for every $\theta \in \underline{T}^{*}$ one has

$$
\rho_{i}\left(d_{h} \theta\right)(X, Y)=d_{h^{i}} \theta(X, Y)+\sum_{k=1}^{i-1} d \theta\left(h^{i-k} X, h^{k} Y\right) .
$$

In particular, every conservation law for $h$ is a conservation law for $h^{i}(i \geq 1)$, and the identity

$$
\rho_{i} d d_{h} f=d d_{h^{i}} f
$$

holds for any function $f \in \mathcal{C}^{\infty}(M)$.

Proof. One has

$$
\left(d_{h} \theta\right)(X, Y)=h X . \theta(Y)-h Y . \theta(X)-\theta([h X, Y]+[X, h Y]-h[X, Y]),
$$


and hence

$$
\begin{aligned}
\rho_{i}\left(d_{h} \theta\right) & =\sum_{k=1}^{i}\left(d_{h} \theta\right)\left(h^{i-k} X, h^{k-1} Y\right) \\
& =\sum_{k=1}^{i}\left(h^{i-k+1} X \cdot \theta\left(h^{k-1} Y\right)-h^{k} Y \cdot \theta\left(h^{i-k} X\right)\right. \\
& \left.-\theta\left(\left[h^{i-k+1} X, h^{k-1} Y\right]+\left[h^{i-k} X, h^{k} Y\right]-h\left[h^{i-k} X, h^{k-1} Y\right]\right)\right) .
\end{aligned}
$$

It is easy to prove by induction the following identity, which holds when $[h, h]=0$ :

$$
h^{i}[X, Y]=\sum_{k=1}^{i} h\left[h^{i-k} X, h^{k-1} Y\right]-\sum_{k=1}^{i-1}\left[h^{i-k} X, h^{k} Y\right] .
$$

Then

$$
\begin{aligned}
\rho_{i}\left(d_{h} \theta\right) & (X, Y) \\
= & \sum_{k=1}^{i} h^{i-k+1} X \cdot \theta\left(h^{k-1} Y\right)-h^{k} Y \cdot \theta\left(h^{i-k} X\right) \\
& -\theta\left(\left[h^{i-k+1} X, h^{k-1} Y\right]+\left[h^{i-k} X, h^{k} Y\right]-h^{i}[X, Y]\right) \\
& +\sum_{k=1}^{i-1} \theta\left(\left[h^{i-k} X, h^{k} Y\right]\right) \\
= & h^{i} X \cdot \theta(Y)+\sum_{k=2}^{i} h^{i-k+1} X \cdot \theta\left(h^{k-1} Y\right)-h^{i} Y \cdot \theta(X) \\
& -\sum_{k=1}^{i-1} h^{k} Y \cdot \theta\left(h^{i-k} X\right) \\
& -\theta\left(\left[h^{i} X, Y\right]+\sum_{k=2}^{i}\left[h^{i-k+1} X, h^{k-1} Y\right]\right)-\theta\left(\left[X, h^{i} Y\right]+\theta\left(h^{i}[X, Y]\right)\right. \\
= & \left(d_{h^{i}} \theta(X, Y)+\sum_{k=2}^{i} d \theta\left(h^{i-k+1} X, h^{k-1} Y\right)=\left(d_{h^{i}} \theta\right)(X, Y)\right. \\
& +\sum_{k=1}^{i-1} d \theta\left(h^{i-k} X, h^{k} Y\right) . \quad \square
\end{aligned}
$$

\subsection{Involutivity of the differential operator $d d_{h}$.}

Proposition 1.2. The differential operator $d d_{h}$ is involutive.

Proof. Since the degree of $d d_{h}$ is 2 , its symbol is a map $\sigma_{o}: S^{2} T^{*} \longrightarrow \wedge^{2} T^{*}$. As in [2] we have

$$
g_{o}:=\operatorname{Ker} \sigma_{o}=\left\{\varphi \in S^{2} T^{*} \mid \varphi(h X, Y)=\varphi(X, h Y) \forall X, Y \in T M\right\} .
$$

Its prolongation is a map $\sigma_{i}: S^{3} T^{*} \longrightarrow T^{*} \otimes \wedge^{2} T^{*}$, and

$$
g_{1}:=\operatorname{Ker} \sigma_{1}=\left\{\psi \in S^{3} T^{*} \mid \psi(X, h Y, Z)=\psi(X, Y, h Z) \forall X, Y, Z \in T M\right\} .
$$


In order to calculate the dimension of these spaces at a point $x \in M$, consider a decomposition of $T_{x} M$ into cyclic subspaces:

$$
T_{x} M=V_{1} \oplus \cdots \oplus V_{s} .
$$

We let $q_{i}:=\operatorname{dim}_{i}$ and suppose that $V_{i}$ are arranged in such a way that $q_{1} \geq q_{2} \geq$ $\cdots \geq q_{s}$, where $q_{i}$ are the degrees of the elementary divisors.

Let $v_{i}^{1}$ be a generator of $V_{i}($ for $i=1, \cdots, s)$ and denote $v_{i}^{\alpha}:=h^{\alpha-1} v_{i}^{1} \quad(\alpha=$ $\left.1, \cdots, q_{i}\right)$. The vectors

$$
\left\{\left(v_{1}^{\alpha_{1}}\right)_{\alpha_{1}=1, \cdots, q_{1}},\left(v_{2}^{\alpha_{2}}\right)_{\alpha_{2}=1, \cdots, q_{2}}, \cdots,\left(v_{s}^{\alpha_{s}}\right)_{\alpha_{s}=1, \cdots, q_{s}}\right\} \equiv\left\{v_{i}^{\alpha_{i}}\right\}_{\substack{i=1 \cdots s \\ \alpha_{i}=1 \cdots q_{i}}}
$$

form a basis of $T_{x} M$ which is called "adapted" to the decomposition into cyclic subspaces. We shall show that, up to a permutation, this is a quasi-regular basis.

Let $\varphi \in g_{o}$; since $\varphi$ is symmetric it suffices to consider $\varphi\left(v_{i}^{\alpha}, v_{j}^{\beta}\right)$ with $i \leq j$. Now, for $j$ fixed, $j=1, \cdots, s$ and $i \geq j$

$$
\varphi\left(v_{i}^{\alpha}, v_{j}^{\beta}\right)=\varphi\left(h^{\alpha-1} v_{i}^{1}, v_{j}^{\beta}\right)=\varphi\left(v_{i}^{1}, h^{\alpha-1} v_{j}^{\beta}\right)
$$

thus the $\varphi\left(v_{i}^{\alpha}, v_{j}^{\beta}\right)$ with $j$ fixed, $j=1, \cdots, s$, and $i \leq j$ can be expressed in terms of the values of $\varphi$ on $\left(v_{i}^{1}, v_{j}^{\gamma}\right)_{\substack{i \leq j \\ \gamma=1 \cdots q_{j}}}$, whose number is $j q_{j}$. Therefore $\operatorname{dimg}_{o}=$ $\sum_{j=1}^{s} j q_{j}$.

In the same way let $\psi \in g_{1} ; \psi$ is symmetric and the $\psi\left(v_{i}^{\alpha}, v_{j}^{\beta}, v_{k}^{\gamma}\right)$ with $k=$ $1, \cdots, s, i \leq j \leq k$, can be expressed in terms of $\varphi\left(v_{i}^{1}, v_{j}^{1}, v_{k}^{\gamma}\right) \underset{\substack{k=1 \ldots s \\ i \leq j \leq k}}{\text {. Therefore }}$

$$
\operatorname{dim} g_{1}=\sum_{k=1}^{s} \frac{k(k+1)}{2} q_{k}
$$

Let $\mathcal{B}=\left\{v_{1}^{1}, v_{2}^{1}, \cdots, v_{s}^{1}, \cdots\right\}$ be a basis of $T_{x} M$ obtained from an adapted basis after transpositions putting the generators $v_{1}^{1}, \cdots, v_{s}^{1}$ in the first $s$ places. We shall show that this basis is quasi-regular.

One has

$$
\operatorname{dim}\left(g_{o}\right)_{v_{1}^{1}}=\operatorname{dim}\left\{\varphi \in g_{o} \mid i_{v_{1}^{1}} \varphi=0\right\}=\operatorname{Card}\left\{v_{i}^{1}, v_{j}^{\alpha}\right\} \begin{gathered}
\substack{j=1 \cdots s \\
i=2 \ldots j \\
\alpha=1 \cdots q_{j}} \\
\alpha=1
\end{gathered}=\sum_{j=1}^{s}(j-1) q_{j}
$$

and, more generally,

$$
\operatorname{dim}\left(g_{o}\right)_{v_{1}^{1} \cdots v_{\alpha}^{1}}=\sum_{j=\alpha}^{s}(j-\alpha) q_{j} \quad\left(\alpha=1, \ldots, q_{j}\right) .
$$

Moreover, $\left(g_{o}\right)_{v_{1}^{1} \cdots v_{s}^{1}}=\{0\}$, and then $\left(g_{o}\right)_{\mathcal{F}}=\{0\}$ for every subset $\mathcal{F}$ of the basis $\mathcal{B}$ which contains $\left\{v_{1}^{1} \cdots v_{s}^{1}\right\}$. Then, if we denote by $\left\{e_{1} \cdots e_{n}\right\}$ the basis $\mathcal{B}(n=$ $\operatorname{dim} M)$, we have

$$
\operatorname{dim}\left(g_{o}\right)+\sum_{i=1}^{n} \operatorname{dim}\left(g_{o}\right)_{e_{1} \cdots e_{i}}=\sum_{\alpha=o}^{s} \sum_{j=\alpha}^{s}(j-\alpha) q_{j}=\sum_{k=1}^{s} \frac{k(k+1)}{2} q_{k}=\operatorname{dim} g_{1},
$$

which shows that $\mathcal{B}$ is quasi-regular. 
1.3. The obstruction space. Let $P=d d_{h}$; we have the following diagram of exact sequences:

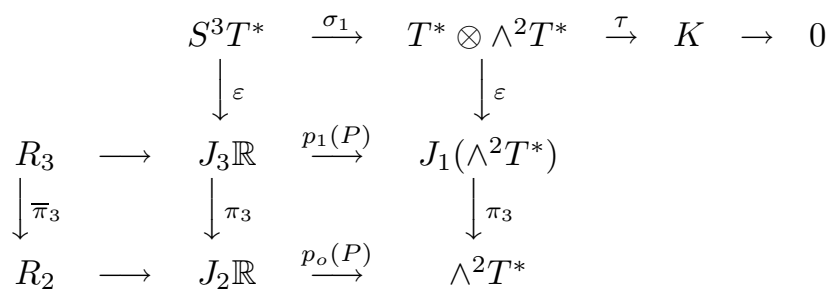

Since $P$ is involutive, proving the formal integrability is equivalent to proving that $\bar{\pi}_{3}$ is onto. For that, we need a "good interpretation" of the obstructions space $K$.

Let us introduce the following notations. $\mathcal{B}=\left\{v_{i}^{\alpha_{i}}\right\}_{\substack{i=1 \ldots s \\ \alpha_{i}=1 \cdots q_{i}}}$ being a basis adapted to a decomposition of $T_{x} M$ in cyclic subspaces, we set

$$
E_{1}:=\operatorname{Span}\left(v_{i}^{1}, v_{2}^{1}, \cdots, v_{s}^{1}\right)
$$

(subspace spanned by the generators) and $E_{\alpha}=h^{\alpha-1}\left(E_{1}\right)\left(\alpha=1, \cdots, q_{1}\right)$.

Let $n_{\alpha}:=\operatorname{dim} E_{\alpha}(c f$. Table 1)

TABLE 1

\begin{tabular}{||c|c|lllllll||}
\hline $\begin{array}{c}\text { dimension } \\
\text { of } V_{i}\end{array}$ & $\begin{array}{c}\text { cyclic } \\
\text { subspaces }\end{array}$ & & & & & & \\
\hline$q_{1}$ & $V_{1}$ & $v_{1}^{1} \stackrel{h}{\longrightarrow}$ & $v_{1}^{2} \stackrel{h}{\longrightarrow}$ & $v_{1}^{3} \stackrel{h}{\longrightarrow} \cdots$ & $\stackrel{h}{\longrightarrow} v_{1}^{\alpha}$ & $\stackrel{h}{\longrightarrow} \cdots$ & $\stackrel{h}{\longrightarrow} v_{1}^{q_{1}}$ \\
$\vdots$ & $\vdots$ & $\vdots$ & & & & & & \\
$q_{i}$ & $V_{i}$ & $v_{i}^{1} \stackrel{h}{\longrightarrow}$ & $v_{i}^{2} \stackrel{h}{\longrightarrow}$ & $v_{i}^{3} \stackrel{h}{\longrightarrow} \cdots$ & $\stackrel{h}{\longrightarrow} v_{i}^{\alpha}$ & $\stackrel{h}{\longrightarrow} v_{i}^{q_{i}}$ & \\
$\vdots$ & $\vdots$ & $\vdots$ & & & & & & \\
$q_{s}$ & $V_{s}$ & $v_{s}^{1} \stackrel{h}{\longrightarrow}$ & $v_{s}^{2} \longrightarrow$ & $\cdots \stackrel{h}{\longrightarrow} v_{s}^{q_{s}}$ & & \\
\hline & & $E_{1}$ & $E_{2}$ & & & & \\
& & $\begin{array}{c}\text { dimension } \\
\text { of } E_{i}\end{array}$ & $n_{1}$ & $n_{2}$ & & & \\
\hline
\end{tabular}

Note that $n_{1}=s=$ number of cyclic subspaces and that $n_{1} \geq n_{2} \geq \cdots \geq n_{q_{1}}$, and $\sum_{\alpha=1}^{q_{1}} n_{\alpha}=n$. When $s=1, n_{1}=n_{2}=\cdots=n_{q_{1}}=1$ and $q_{1}=n$, we have the cyclic case.

Proposition 1.3. Consider the exact sequence $S^{2} T^{*} \stackrel{\sigma_{o}}{\longrightarrow} \wedge^{2} T^{*} \longrightarrow K_{o} \longrightarrow 0$, where $K_{o}:=\operatorname{CoKer} \sigma_{o}=\frac{\wedge^{2} T^{*}}{I m \sigma_{o}}$. One has

$$
\operatorname{dim} K_{o}=\sum_{j=1}^{s}(j-1) q_{j}=\sum_{\alpha=1}^{q_{1}} \frac{n_{\alpha}\left(n_{\alpha}-1\right)}{2} .
$$

Indeed,

$$
\operatorname{dim} K_{o}=\operatorname{dim} \wedge^{2} T^{*}-\operatorname{dim} S^{2} T^{*}+\operatorname{dim} g_{o}=-n+\sum_{j=1}^{s} j q_{j} .
$$


Now $n=\sum_{j=1}^{s} q_{j}$ and $\operatorname{dim} K_{o}=\sum_{j=1}^{s}(j-1) q_{j}$. On the other hand, let $\varphi(\ell)$ be the number of subspaces $E_{i}$ whose dimension is $\ell$. If one computes the number of skew-symmetric matrices built on every space $E_{\alpha}\left(\alpha=1, \cdots, q_{1}\right)$, one has

$$
\sum_{\alpha=1}^{q_{1}} \frac{n_{\alpha}\left(n_{\alpha}-1\right)}{2}=\sum_{\ell=1}^{s} \frac{\ell(\ell-1)}{2} \varphi(\ell) .
$$

If one computes the columns of Table 1 , it is not difficult to prove that $q_{\alpha}=$ $\varphi(\alpha)+\varphi(\alpha+1)+\cdots+\varphi(s)$. Thus:

$$
\begin{aligned}
\operatorname{dim} K_{o} & =\sum_{j=1}^{s}(j-1) q_{j}=\sum_{q=1}^{s} \sum_{\ell=j}^{s} \varphi(\ell) \\
& =\sum_{\ell=1}^{s} \varphi(\ell) \sum_{\alpha-1}^{\ell}(\alpha-1)=\sum_{\ell=1}^{s} \varphi(\ell) \frac{\ell(\ell-1)}{2}=\sum_{\alpha=1}^{q_{1}} \frac{n_{\alpha}\left(n_{\alpha}-1\right)}{2} .
\end{aligned}
$$

Taking into account Proposition 1.3 and the identity $n=\sum_{j=1}^{s} q_{j}$, a standard computation proves that

$$
\sum_{j=1}^{s} \frac{(j-1)(j+2)}{2} q_{j}=s \sum_{\alpha=1}^{q_{1}} \frac{n_{\alpha}\left(n_{\alpha}-1\right)}{2}-\sum_{j=1}^{s} \frac{(j-1)(2 s-j-2)}{2} q_{j} .
$$

From this one can easily deduce:

Proposition 1.4. $K$ is isomorphic to a subspace of codimension

$$
\sum_{j=1}^{s} \frac{(j-1)(2 s-j-2)}{2} q_{j}
$$

in $\mathcal{E}=\wedge^{3} T^{*} \times \wedge^{3} T^{*} \times\left(E_{1}^{*} \otimes K_{o}\right)$.

\section{THE NILPOTENT CASE}

2.1. The exact sequence of the symbol. In this section, we suppose that $h$ is nilpotent of order $p, p \geq 2$, the case $p=1$ being trivial. According to the notations of paragraph 1.3, $q_{1}=p$ is the degree of the minimal polynomial of $h$.

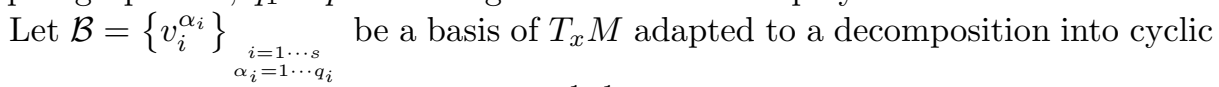
subspaces; denote (as in 1.3) $v_{i}^{\alpha}:=h^{\alpha-1} v_{i}^{1}$ and $v_{i}^{\alpha}=0$ for $\alpha \leq 0$. It appears from Table 1 that the vectors $v_{1}^{q_{1}}, \cdots, v_{s}^{q_{s}}$ (the last of each line) span Ker $h$; in the same way the last two of every line span Ker $h^{2}$, and so on. Then if we set

$$
\begin{aligned}
& F_{1}=\operatorname{Span}\left(v_{1}^{q_{1}}, v_{2}^{q_{2}}, \cdots, v_{s}^{q_{s}}\right) \\
& F_{2}=\operatorname{Span}\left(v_{1}^{q_{1}-1}, v_{2}^{q_{2}-1}, \cdots, v_{s}^{q_{s}-1}\right) \\
& \vdots \\
& F_{\alpha}=\operatorname{Span}\left(v_{1}^{q_{1}-\alpha+1}, v_{2}^{q_{2}-\alpha+1}, \cdots, v_{s}^{q_{s}-\alpha+1}\right) \quad\left(\alpha=1, \cdots, q_{1}\right)
\end{aligned}
$$

we have

$$
\operatorname{Kerh}^{\alpha}=F_{1} \oplus F_{2} \oplus \cdots \oplus F_{\alpha} .
$$

One has $\operatorname{dim} F_{\alpha}=n_{\alpha}$, and therefore

$$
n_{\alpha}=\operatorname{dim} \frac{\operatorname{Ker} h^{\alpha}}{\operatorname{Ker} h^{\alpha-1}}
$$


because $F_{\alpha}$ is isomorphic to $E_{\alpha}$. Indeed a basis of $E_{\alpha}$ is formed by the nonzero vectors $\left\{v_{i}^{\alpha}\right\}_{i=1 \ldots s}$ and a basis of $F_{\alpha}$ by the nonzero vectors $\left\{v_{1}^{q_{i}-\alpha+1}\right\}_{i=1 \ldots s}$. Now $v_{i}^{\alpha} \neq 0$ if and only if $1 \leq \alpha \leq q_{i}$; on the other hand, $v_{i}^{q_{i}-\alpha+1} \neq 0$ if and only if $1 \leq q_{i}-\alpha+1 \leq q_{i}$, i.e. $1 \leq \alpha \leq q_{i}$. Thus the bases $E_{\alpha}$ and $F_{\alpha}$ have the same cardinality.

Proposition 2.1. Suppose that $h$ is nilpotent of order $p\left(p=q_{1}\right)$ and let, for $\alpha=1, \cdots, p, \widetilde{\rho}_{\alpha}: \wedge^{2} T^{*} \longrightarrow \wedge^{2}\left(\operatorname{Ker} h^{\alpha}\right)^{*}$ be the map defined by

$$
\left(\widetilde{\rho}_{\alpha} \Omega\right)(U, V)=\sum_{k=1}^{\alpha} \Omega\left(h^{\alpha-k} U, h^{k-1} V\right) \quad \forall U, V \in \operatorname{Ker} h^{\alpha}
$$

(that is, $\widetilde{\rho}={ }^{t} j_{\alpha} \circ \rho_{\alpha}$, where $j_{\alpha}: \operatorname{Ker} h^{\alpha} \longrightarrow T$ is the canonical injection). One has $\widetilde{\rho}_{\alpha} \circ \sigma_{0}=0$ and $K_{0} \simeq \operatorname{Im} \widetilde{\rho}_{1} \times \cdots \times \operatorname{Im} \widetilde{\rho}_{p}$. In other words, if we define $\widetilde{\rho}: \wedge^{2} T^{*} \longrightarrow \wedge^{2}(\operatorname{Ker} h)^{*} \times \cdots \times \wedge^{2}\left(\operatorname{Ker} h^{p}\right)^{*}$ by $\widetilde{\rho}:=\widetilde{\rho}_{1} \times \cdots \times \widetilde{\rho}_{p}$, the sequence

$$
S^{2} T^{*} \stackrel{\sigma_{0}}{\longrightarrow} \wedge^{2} T^{*} \stackrel{\widetilde{\rho}}{\longrightarrow} \operatorname{Im} \widetilde{\rho} \longrightarrow 0
$$

is exact.

Proof. One can easily see that

$$
\left(\widetilde{\rho}_{\alpha} \circ \sigma_{o}\right)(\varphi)(U, V)=\varphi\left(h^{\alpha} U, V\right)-\varphi\left(U, h^{\alpha} U\right)=0
$$

for $\varphi \in S^{2} T^{*}$, since $U, V \in \operatorname{Kerh} h^{\alpha}$. Then $\widetilde{\rho} \circ \sigma_{o}=0$ and $\operatorname{rk} \widetilde{\rho} \leq \operatorname{dim} K_{o}$. We need only to show that $\operatorname{rk} \tilde{\rho} \geq \operatorname{dim} K_{o}$.

Let $S$ be the system defined by $\widetilde{\rho} \Omega=0$, and $S^{\prime}$ the subsystem defined by

$$
\left.\widetilde{\rho}_{\alpha} \Omega\right|_{F_{\alpha} \wedge F_{\alpha}}=0 \quad(\alpha=1, \cdots, p) .
$$

One has $\operatorname{rk} S^{\prime} \leq \operatorname{rk} S$. On the other hand, if we use a basis adapted to the decomposition $T M=F_{1} \oplus \cdots \oplus F_{p}, S^{\prime}$ can be written as

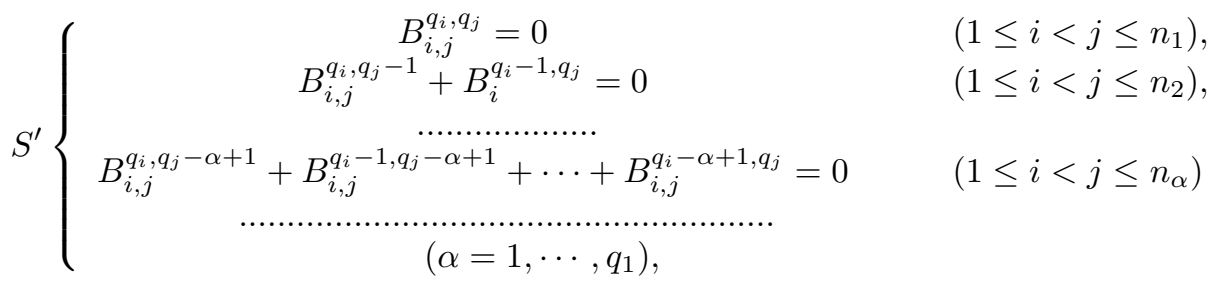

where

$$
B_{i j}^{r s}=\Omega\left(v_{i}^{r}, v_{j}^{s}\right) .
$$

Noting that the variables $B_{i j}^{r s}$ do not appear formally in the system if and only if $v_{i}^{r}=0$ or $v_{j}^{s}=0$ (that is: if and only if $r \leq 0$ or $s \leq 0$ ), it is not difficult to show (cf. Table 1 ) that $n_{\alpha} \geq i$ implies $q_{i} \geq \alpha$. Therefore, in each equation all the variables appear, and then each equation appears in the system. Hence $S^{\prime}$ contains $\sum_{\alpha=1}^{q_{1}} \frac{n_{\alpha}\left(n_{\alpha}-1\right)}{2}=\operatorname{dim} K_{o}$ equations. On the other hand, all these equations are independent because each variable appears in one and only one equation. Therefore $\operatorname{rk} S^{\prime}=\operatorname{dim} K_{o}$; then $\operatorname{rk} S \geq \operatorname{dim} K_{o}$, and then $\operatorname{rk} S=\operatorname{dim} K_{o}$. 
Remark 1. In particular, we showed that

$$
\tilde{\rho}_{\alpha} \Omega=0 \quad \forall \alpha=1, \cdots,\left.p \Longleftrightarrow \widetilde{\rho}_{\alpha} \Omega\right|_{F_{\alpha} \wedge F_{\alpha}}=0 \quad \forall \alpha=1, \cdots, p .
$$

In the same way, it can be shown that

$$
\tilde{\rho}_{\alpha} \Omega=0 \quad \forall \alpha=1, \cdots,\left.r \Longleftrightarrow \tilde{\rho}_{\alpha} \Omega\right|_{F_{\alpha} \wedge F_{\alpha}}=0 \quad \forall \alpha=1, \cdots, r .
$$

\subsection{The space of second order formal solutions.}

Definition 2.1. Let $h \in \underline{T^{*} \otimes T}$; we define the map $C_{h}: \wedge^{2} \operatorname{Ker} h \longrightarrow \operatorname{Im} h$ by $C_{h}(U, V)=h[U, V]$.

It can be easily shown that $C_{h} \in \wedge^{2}(\operatorname{Ker} h)^{*} \otimes \operatorname{Im} h$ (that is: $C_{h}$ is a tensor), and it is obvious that $C_{h}=0$ if and only if Kerh is involutive.

We denote by $\mathbb{K}_{i}$ the subspace of $\operatorname{Im} h^{i}$ spanned by the image of $C_{h^{i}}$. Note that $h^{2 i}[U, V]=0$ for all $U, V \in \operatorname{Ker} h^{i}$ because $\left[h^{i}, h^{i}\right]=0$, and therefore $\mathbb{K}_{i} \subset \operatorname{Ker} h^{i}$; thus

$$
\mathbb{K}_{i} \subset \operatorname{Im} h^{i} \cap \operatorname{Ker} h^{i} .
$$

Proposition 2.2. Suppose that $h$ is nilpotent of order $p$, and let $R_{2}$ be the space of second order formal solutions of the differential operator $d d_{h}$ at a point $x_{o} \in M$. If $\bar{\pi}_{2}: R_{2} \longrightarrow T_{x_{o}}^{*}$ is the restriction $\bar{\pi}_{2}:=\left.\pi_{2}\right|_{R_{2}}$, of the natural surjection $\pi_{2}$ : $\left(J_{2}\right)_{x_{o}} \mathbb{R} \longrightarrow\left(J_{1}\right)_{x_{o}} \mathbb{R} \simeq T_{x_{o}}^{*}$, then at any point of $M$

$$
\bar{\pi}_{2}\left(R_{2}\right)=\mathbb{K}_{1}^{o} \cap \cdots \cap \mathbb{K}_{p}^{o}
$$

where $\mathbb{K}_{i}^{o}$ is the annihilator of the space $\mathbb{K}_{i}$. In other words, a form $\omega \in T_{x_{o}}^{*}$ can be lifted to a second order solution if and only if

$$
\omega \circ C_{h^{i}}=0 \quad(i=1, \ldots, p-1) .
$$

Proof. A straightforward calculation shows that

$$
d d_{h} f=d f \circ(\mathcal{A} \nabla h)-\sigma_{o}(\nabla d f)
$$

holds for every functions $f$, where $\nabla$ is a linear connection without torsion on $M$ and $\mathcal{A}$ is the alternation operator : $(\mathcal{A} \varphi)(X, Y)=\varphi(X, Y)-\varphi(Y, X)$. Now, taking into account Proposition 2.1 one has $\widetilde{\rho}_{\alpha}(d f \circ \mathcal{A} \nabla h)=\widetilde{\rho}_{\alpha} d d_{h} f=-d f \circ C_{h^{\alpha}}$. Indeed, according to Proposition 1.1, $\rho_{\alpha} d d_{h} f=d d_{h^{\alpha}} f$, and hence

$$
\begin{aligned}
\widetilde{\rho}_{\alpha}\left(d d_{h} f\right)(X, Y) & =\left(X . h^{\alpha} Y-Y \cdot h^{\alpha} Y-h^{\alpha}[X, Y]\right) \cdot f \\
& =-h^{\alpha}[X, Y] . f=-\left(d f \circ C_{h^{\alpha}}\right)(X, Y)
\end{aligned}
$$

for each $X, Y \in \operatorname{Ker}^{\alpha}$. Then $\forall \omega_{x_{o}} \in T_{x_{o}}^{*}$ one has

$$
\tilde{\rho}_{\alpha}\left(\omega_{x_{o}} \circ \mathcal{A} \nabla h\right)=-\omega_{x_{o}} \circ C_{h^{\alpha}} .
$$

Let us take $\omega_{x_{o}} \in\left(\mathbb{K}_{1}^{o}\right)_{x_{o}} \cap \cdots \cap\left(\mathbb{K}_{p-1}^{o}\right)_{x_{o}}$; i.e., such that $\omega_{x_{o}} \circ C_{h^{\alpha}}=0, \forall \alpha=$ $1, \cdots, p$. One has $\widetilde{\rho}_{\alpha}\left(\omega_{x_{o}} \circ \mathcal{A} \nabla h\right)=0 \forall \alpha=1, \cdots, p$; that is to say, $\widetilde{\rho}\left(\omega_{x_{o}} \circ \mathcal{A} \nabla h\right)=$ 0 . Since Ker $\widetilde{\rho}=\operatorname{Im} \sigma_{o}$, there exists $\theta_{x_{o}} \in S^{2} T_{x_{o}}^{*}$ for which $\omega_{x_{o}} \circ \mathcal{A} \nabla h=\sigma_{o}\left(\theta_{x_{o}}\right)$. Let $f$ be a germ of a function at $x_{o}$ such that $\omega_{x_{o}}=(d f)_{x_{o}}$ and $\theta_{x_{o}}=(\nabla d f)_{x_{o}}$ (which exists, as can be verified easily in a normal coordinate system). We have

$$
(d f)_{x_{o}} \circ \mathcal{A} \nabla h-\sigma_{o}(\nabla d f)_{x_{o}}=0,
$$


i.e. $\left(d d_{h} f\right)_{x_{o}}=0$. This proves that $\omega_{x_{o}}$ can be lifted to a second order solution.

The opposite assertion is obvious from the identity $\widetilde{\rho}_{\alpha}\left(d d_{h} f\right)=-d f \circ C_{h^{\alpha}}$.

Corollary 2.1. Suppose that $h$ is nilpotent. Then any $\omega \in T_{x_{o}}^{*}$ such that $i_{h} \omega=0$ (i.e. $\omega \in \operatorname{Ker}^{*}$ ) can be lifted to a second order formal solution at $x_{o}$. In particular

$$
\bar{\pi}_{2} R_{2} \neq\{0\} .
$$

Indeed, $\mathbb{K}_{\alpha} \subset \operatorname{Im} h^{\alpha} \subset \operatorname{Im} h, \forall \alpha=1, \cdots, p$; then $\mathbb{K}_{\alpha}^{o} \supset$ Ker $h^{*}, \forall \alpha=1, \cdots, p$ and hence $\bar{\pi}_{2} R_{2} \supset \operatorname{Ker}^{*}$. Now Ker $h^{*} \neq\{0\}$ because $h$ is nilpotent; then $\bar{\pi}_{2}\left(R_{2}\right) \neq$ $\{0\}$.

Corollary 2.2. Let $h^{p}=0$. Then every form in $T^{*}$ can be lifted to a second order formal solution if and only if $\operatorname{Ker} h, \operatorname{Ker} h^{2}, \cdots, \operatorname{Ker}^{p-1}$ are involutive.

Remark. There exist nilpotent tensor fields $h$ of type (1-1) satisfying $[h, h]=0$ for which Kerh is not involutive. Consider, for example, the field $h$ on $\mathbb{R}^{6}$ defined by

$$
h=d x^{1} \otimes \frac{\partial}{\partial x^{2}}+d x^{2} \otimes \frac{\partial}{\partial x^{3}}+d x^{4} \otimes \frac{\partial}{\partial x^{5}}+d x^{6} \otimes\left(-x^{4} \frac{\partial}{\partial x^{2}}-x^{5} \frac{\partial}{\partial x^{3}}\right) .
$$

One can easily verify that $h^{3}=0,[h, h]=0$, and $\mathbb{K}_{1}$ is spanned by $\frac{\partial}{\partial x^{3}}$; thus $\mathbb{K}_{1} \neq\{0\}$.

\subsection{The exact sequence of the first prolongation.}

Proposition 2.3. Suppose that $h$ is nilpotent of order $p(p \geq 2)$ and define the map

$$
\tau: T^{*} \otimes \wedge^{2} T^{*} \longrightarrow \wedge^{3} T^{*} \times \wedge^{3} T^{*} \times\left(E_{1}^{*} \otimes K_{0}\right) \quad\left(\tau=\tau_{1} \times \tau_{2} \times \tau_{3}\right)
$$

by

$$
\begin{gathered}
\left(\tau_{1} \omega\right)(X, Y, Z):=\sum_{\operatorname{cycl}(X, Y, Z)} \omega(X, Y, Z),\left(\tau_{2} \omega\right)(X, Y, Z):=\sum_{\operatorname{cycl}(X, Y, Z)} \omega(h X, Y, Z), \\
\left(\tau_{3}^{\alpha} \omega\right)\left(X_{1}, Y_{\alpha}, Z_{\alpha}\right):=\sum_{k=1}^{\alpha} \omega\left(X_{1}, h^{\alpha-k} Y_{\alpha}, h^{k-1} Y_{\alpha}\right)
\end{gathered}
$$

$\forall X, Y, Z \in T, \forall X_{1} \in E_{1}, \forall Y_{\alpha}, Z_{\alpha} \in \operatorname{Ker} h^{\alpha},\left(\alpha_{1}, \cdots p\right)$. Then $\tau \circ \sigma_{1}=0$ and $K \simeq$ $\operatorname{Im} \tau$. In other words, if $\bar{\tau}: T^{*} \otimes \wedge^{2} T^{*} \rightarrow K, \omega \mapsto \tau(\omega)$, then the sequence

$$
S^{3} T^{*} \stackrel{\sigma_{1}}{\longrightarrow} T^{*} \otimes \wedge^{2} T^{*} \stackrel{\bar{\tau}}{\longrightarrow} K \longrightarrow 0
$$

is exact.

Proof. It is a straightforward verification that $\tau_{1} \circ \sigma_{1}=\tau_{2} \circ \sigma_{1}=0$ (cf. [2]). On the other hand $\tau_{3}^{\alpha}={ }^{t} i \otimes \widetilde{\rho}_{\alpha}$, where $i: E_{1} \longrightarrow T$ is the natural inclusion. Then

$$
\tau_{3}^{\alpha} \circ \sigma_{1}=\left({ }^{t} i \otimes \widetilde{\rho}_{\alpha} \circ \sigma_{1}\right)=\left({ }^{t} i \otimes \widetilde{\rho}_{\alpha}\right) \circ\left(i d_{T^{*}} \otimes \sigma_{o}\right)=0
$$

because $\widetilde{\rho}_{\alpha} \circ \sigma_{o}=0$ (cf. 2.1); therefore $\tau \circ \sigma_{1}=0$.

We have to prove that the rank of the system $\tau(\omega)=0$ is equal to the dimension of $K$. Consider the system $S$ defined by $\tau(\omega)=0$.

Lemma 2.1. The system $S$ is equivalent to the system

$$
S^{\prime}\left\{\begin{array}{l}
\omega(X, Y, Z)+\omega(Y, Z, X)+\omega(Z, X, Y)=0, \\
\omega(h X, Y, Z)+\omega(h Y, Z, X)+\omega(h Z, X, Y)=0, \\
\omega\left(X_{1}, h^{r-1} Y_{r}, Z_{r}\right)+\omega\left(X_{1}, h^{r-2} Y_{r}, h Z_{r}\right)+\cdots+\omega\left(X_{1}, Y_{r}, h^{r-1} Z_{r}\right)=0
\end{array}\right.
$$


$\forall X, Y, Z \in T, \forall X_{1} \in E_{1}, \forall Y_{r}, Z_{r} \in F_{r}, r=1, \cdots, q_{1}=p$, where the $F_{i}$ are the subspaces defined in section 2.1

Indeed $\tau_{3}^{r} \omega=0$ if and only if $\forall X_{1} \in E_{1} \widetilde{\rho}_{r}\left(i_{X_{1}} \omega\right)=0$, which is equivalent to $\left.\widetilde{\rho}_{r}\left(i_{X_{1}} \omega\right)\right|_{F_{r} \wedge F_{r}}=0$ according to Remark 1. Note that the system $S^{\prime}$ contains $\frac{n(n-1)(n-2)}{3}+s \sum_{r=1}^{q_{1}} \frac{n_{r}\left(n_{r}-1\right)}{2}=\operatorname{dim} \mathcal{E}$ equations. Since the codimension of $K$ in $\mathcal{E}$ is $\sum_{j=1}^{s} \frac{(j-1)(2 s-j-2)}{2} q_{j}$ (cf. 1.4$)$ we have only to prove that there are exactly $\sum_{j=1}^{s} \frac{(j-1)(2 s-j-2)}{2} q_{j}$ independent relations in the system $S^{\prime}$.

Let $\left\{v_{i}^{\alpha}\right\}_{\substack{i=1 . . \ldots, q_{1} \\ \alpha=1, \ldots, q_{1}}}$ be a basis adapted to the decomposition into cyclic subspaces, and note that $v_{j}^{\beta} \in F_{r}\left(r=1, \cdots, q_{1}\right)$ and $v_{j}^{\beta} \neq 0$ if and only if $\beta=q_{j}+1-r$. Let us set

$$
C_{i j k}^{\alpha \beta \gamma}:=\omega\left(v_{i}^{\alpha}, v_{j}^{\beta}, v_{k}^{\gamma}\right) .
$$

Then $S^{\prime}$ is equivalent to the system

$$
\left(S^{\prime}\right) \begin{cases}L_{i j k}^{\alpha \beta \gamma} & C_{i j k}^{\alpha \beta \gamma}+C_{j k i}^{\beta \gamma \alpha}+C_{k i j}^{\gamma \alpha \beta}=0, \\ M_{i j k}^{\alpha \beta \gamma} & C_{i j k}^{\alpha+1 \beta \gamma}+C_{j k i}^{\beta+1} \gamma \alpha+C_{k i j}^{\gamma+1 \alpha \beta}=0, \\ N_{i j k}^{1 q_{j}-r+1 q_{k}-r+1} & C_{i j k}^{1} q_{j} q_{k}-r+1+C_{i j k}^{1 q_{j}-1 q_{k}-r+2} \\ & +\cdots+C_{i j k}^{1 q_{j}-r+1 q_{k}}=0 .\end{cases}
$$

Let $\theta$ be an integer such that $3 \leq \theta \leq q_{i}+q_{j}+q_{k}+1$. It is not difficult to show that the variables $C_{i j k}^{\alpha \beta \gamma}$ with $\alpha+\beta+\gamma=\theta$ appear only in the following equations:

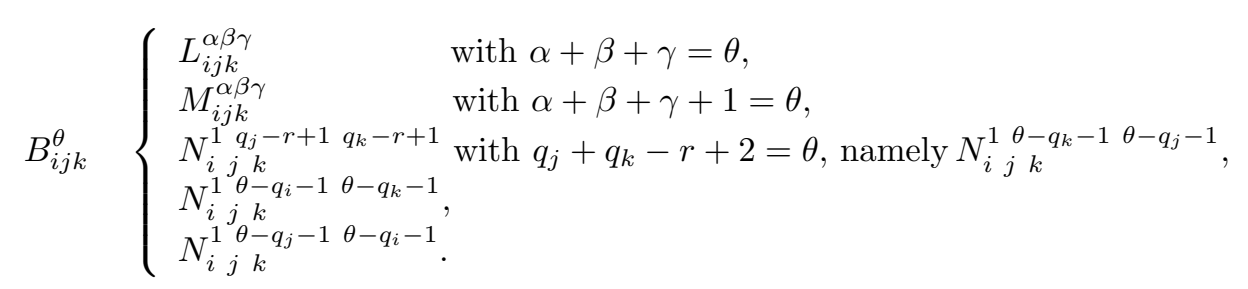

Then, if $B_{i j k}^{\theta}$ denote the subsystem of these equations, all these subsystems are independent. Hence, the rank of the system $S^{\prime}$ is the sum of the ranks of the blocks $B_{i j k}^{\theta}$.

Lemma 2.2. The equations in each block $B_{i j k}^{\theta}$ are all independent, except for those in the blocks $B_{i j k}^{\theta}$ such that

$$
2+q_{i} \leq \theta \leq 1+q_{i}+q_{j}+q_{k} \quad \text { and } \quad 1 \leq i<j<k \leq s,
$$

whose equations are related exactly by one linear relation.

The proof of this lemma is very technical; we give it in Appendix 1.

According to this lemma, the corank of $S^{\prime}$ is equal to the number of blocks $B_{i j k}^{\theta}$ satisfying the condition $(*)$ of the lemma. Now, for every fixed $i, j, k$ such that $1 \leq i<j<k \leq s$ there are $q_{j}+q_{k}$ blocks for which $2+q_{i} \leq \theta \leq 1+q_{i}+q_{j}+q_{k}$. On the whole there are $\sum_{\ell=1}^{s}\left(a_{\ell}+b_{\ell}\right) q_{\ell}$ independent linear relations, where:

$$
\begin{gathered}
a_{\ell}=\text { number of }(i, \ell, k) \text { with } 1 \leq i<\ell<k \leq s=(\ell-1)(s-\ell), \\
b_{\ell}=\text { number of }(i, j, \ell) \text { with } 1 \leq i<j<\ell \leq s=\left(\begin{array}{c}
2 \\
\ell-1
\end{array}\right)=\frac{(\ell-1)(\ell-2)}{2} .
\end{gathered}
$$


Then the number of linear independent relations of the system $S^{\prime}$ is

$$
\sum_{\ell=1}^{s}\left[(\ell-1)(s-\ell)+\frac{(\ell-1)(\ell-2)}{2}\right] q_{\ell}
$$

i.e. $\sum_{j=1}^{s} \frac{(j-1)(2 s-j-2)}{2} q_{j}$, and this completes the proof of Proposition 2.3.

2.4. Formal integrability of $d d_{h}$ in the nilpotent case. In this section we prove the following theorem:

Theorem 2.1. Suppose that $h$ is nilpotent of order $p \geq 2$ with $[h, h]=0$, and define, as in section 2.2, the $\operatorname{map} C_{\alpha}^{*}: T_{x_{o}}^{*} \rightarrow \wedge^{2}\left(\operatorname{Ker}^{\alpha}\right)^{*}$ by

$$
C_{\alpha}^{*}(\omega)(U, V):=\omega\left(C_{\alpha}(U, V)\right) .
$$

Then the differential operator $d d_{h}$ is formally integrable at $x_{o}$ if and only if for every second order solution $F_{o}$ at $x_{o}$

$$
\left(p_{1} C_{\alpha}^{*}\right)\left(F_{o}\right)=0 \quad(\alpha=1, \ldots, p),
$$

where $p_{1} C_{\alpha}^{*}$ denotes the first prolongation of $C_{\alpha}^{*}$ (we identified $T_{x_{o}}^{*}$ with $J_{1, x_{o}} \mathbb{R}$ ).

According to the results of the preceding sections, we have to show that for any linear connection $\nabla$ on $M$, one has $\tau\left(\nabla d d_{h} f\right)_{x_{o}}=0$ when $\left(d d_{h} f\right)_{x_{o}}=0$. As in [2] one can see easily that $\tau_{1}\left(\nabla d d_{h} f\right)_{x_{o}}=\left(d^{2} d_{h} f\right)_{x_{o}}=0$ and $\tau_{2}\left(\nabla d d_{h} f\right)_{x_{o}}=$ $-\left(d_{h}^{2} d f\right)_{x_{o}}=0$ when $\left(d d_{h} f\right)_{x_{o}}=0$.

On the other hand,

$$
\begin{aligned}
\tau_{3}^{\alpha}\left(\nabla d d_{h} f\right)_{x_{o}}\left(X, Y_{\alpha}, Z_{\alpha}\right) & =\tilde{\rho}_{\alpha}\left(\nabla_{X} d d_{h} f\right)_{x_{o}}\left(Y_{\alpha}, Z_{\alpha}\right) \\
& =\sum_{k=1}^{\alpha}\left(\nabla_{X} d d_{h} f\right)_{x_{o}}\left(h^{\alpha-k} Y_{\alpha}, h^{k-1} Z_{\alpha}\right)
\end{aligned}
$$

for $X \in E_{1}$ and $Y_{\alpha}, Z_{\alpha} \in \operatorname{Ker} h^{\alpha}$. Now, taking into account that $\left(d d_{h} f\right)_{x_{o}}=0$, this expression is equal to

$$
\begin{aligned}
\sum_{k=1}^{\alpha} \nabla_{X_{x_{o}}} \cdot\left[\left(d d_{h} f\right)\left(h^{\alpha-k} Y_{\alpha}, h^{k-1} Z_{\alpha}\right)\right] & =\nabla_{X_{x_{o}}} \cdot\left[\left(\widetilde{\rho}_{\alpha} d d_{h} f\right)\left(Y_{\alpha}, Z_{\alpha}\right)\right] \\
& =-\nabla_{X_{x_{o}}} \cdot\left(d f \circ C_{\alpha}\right)\left(Y_{\alpha}, Z_{\alpha}\right)=0 .
\end{aligned}
$$

A particular case is when every 1-form on a open set can be extended in a germ of conservation laws (this is the case of "completely integrable systems"). One has:

Corollary 2.3. Suppose that $h$ is nilpotent of order $p(p \geq 2)$, analytic and such that $[h, h]=0$. Fix $x_{o} \in M$. Then there exists a neighborhood $U$ of $x_{o}$ such that any $x \in U$ admits a "complete system" of conservation laws (i.e. every $\omega_{o} \in T_{x}^{*}$ can be prolonged in a germ of conservation laws) if and only if $\operatorname{Ker} h, \operatorname{Ker} h^{2}, \cdots, \operatorname{Ker} h^{p-1}$ are involutive.

Indeed, these conditions are required in order to lift arbitrary initial conditions to second order solutions (cf. 2.2). On the other hand, if they hold, then second order solutions can be lifted to formal solutions (because $C_{h^{\alpha}}=0$ for any $\alpha=1, \ldots, p-1$ ) which actually are analytic according to the convergence theorem (cf. Appendix 2, Theorem 1). 
Corollary 2.4. (Normal form for $h$ ). Let $h$ be nilpotent of order $p$, analytic, satisfying $[h, h]=0$, and suppose that $\operatorname{Ker} h, \operatorname{Ker} h^{2}, \cdots, \operatorname{Ker}^{p-1}$ are involutive. If $x_{o} \in M$, then there exist local coordinates $\left(x^{1} \cdots x^{n}\right)$ in a neighborhood of $x_{o}$ for which $h$ has the form

$$
\left(\begin{array}{cccc}
J_{q_{1}} & & & \\
& J_{q_{2}} & & \\
& & \ddots & \\
& & & J_{q_{s}}
\end{array}\right)
$$

where the $J_{q_{k}}$ are $q_{k}$-dimensional Jordan blocks.

In particular, the G-structure associated to $h$ is integrable.

Indeed, let $T_{x_{o}}^{*}=W_{1} \oplus \cdots \oplus W_{s}$ be a decomposition into cyclic subspaces associated to $h^{*}$, and consider a generator $\omega_{1}$ of $W_{1}$. According to Corollary 2.2 there exists a conservation law $\widetilde{\omega}_{1}$ for which $\left(\widetilde{\omega}_{1}\right)_{x_{o}}=\omega_{1}$, and then a function $x_{1}$ such that $\widetilde{\omega}_{1}=d x_{1}$. Since $h^{*} \widetilde{\omega}$ is a conservation law, there exists (locally) a function $x_{2}$ such that $h^{*} \widetilde{\omega}=d x_{2}$. Now

$$
\left(d x_{1} \wedge d x_{2}\right)_{x_{o}}=\left(\widetilde{\omega}_{1} \wedge h^{*} \widetilde{\omega}_{2}\right)_{x_{o}}=\omega_{1} \wedge h^{*} \omega_{2} \neq 0 .
$$

In the same way $h^{* 2} \widetilde{\omega}_{1}, \cdots, h^{* q_{1}-1} \widetilde{\omega}_{1}$ are conservation laws $\left(q_{1}=\operatorname{dim} W_{1}\right)$; then there exist functions $x_{3}, \cdots, x_{q_{1}}$ such that $d x_{j}={ }^{t} h^{j-1} \widetilde{\omega}_{1}$. Clearly,

$$
\left(d x_{1} \wedge \cdots \wedge d x_{q_{1}}\right)_{x_{o}}=\omega_{1} \wedge h^{*} \omega_{1} \wedge \cdots \wedge h^{* q_{1}-1} \omega_{1} \neq 0
$$

and $\left(x_{1}, \cdots, x_{q_{1}}\right)$ is a coordinate system in which $\left.h^{*}\right|_{W_{1}}$ takes the form

$$
\left(\begin{array}{cccc}
0 & \cdots & \cdots & 0 \\
1 & \ddots & & \vdots \\
& \ddots & \ddots & 0 \\
0 & \cdots & 1 & 0
\end{array}\right)
$$

Repeating this construction on the other cyclic subspaces, we obtain the matrix of $h^{*}$ in the local coordinates $\left(x_{1} \cdots x_{n}\right)$; its transpose is the matrix of $h$ in this coordinate system.

\section{Reduction to the nilpotent CASE}

3.1. The case where the minimal polynomial is $(X-\lambda)^{p}$. In this subsection we suppose that $h$ has only one eigenvalue $\lambda$, of multiplicity $n$. Let $(X-\lambda)^{p}$ be the minimal polynomial of $h$. The case $p=1$ being trivial ${ }^{1}$, we suppose that $p \geq 2$. Note that the endomorphism $g:=h-\lambda i d$ is nilpotent. Since $d d_{g} f=d d_{h} f-d \lambda \wedge d f$, $d d_{g}$ and $d d_{h}$ differ by a first order differential operator. It follows that if $\sigma_{o}^{\lambda}$ and $\sigma_{1}^{\lambda}$ denote the symbols of $d d_{g}=d d_{h-\lambda i d}$, and its first prolongation, and $K_{o}^{\lambda}:=$ Coker $\sigma_{o}^{\lambda}, K^{\lambda}:=$ Coker $\sigma_{1}^{\lambda}$, then $\sigma_{o}^{\lambda}=\sigma_{o}, \sigma_{1}^{\lambda}=\sigma_{1}, K_{o}^{\lambda}=K_{o}$ and $K^{\lambda}=K$. Then some of results of the preceding sections are still valid, in particular Propositions 1.2-1.4. Propositions 2.1, 2.3 still hold with convenient adaptations, i.e. replacing $\widetilde{\rho}_{\alpha}$ by

$$
\left(\widetilde{\rho}_{\alpha}^{\lambda} \Omega\right)(U, V):=\sum_{k=1}^{\alpha} \Omega\left((h-\lambda i d)^{\alpha-k} U,(h-\lambda i d)^{k-1} V\right)
$$

\footnotetext{
${ }^{1}$ In this case $d d_{h} f=d \lambda \wedge d f$, and $d f$ is a conservation law if and only if $f$ and $\lambda$ are functionally dependent. Then there exists a complete system of conservation laws if and only if $n=1$.
} 
$\forall U, V \in \operatorname{Ker}(h-\lambda i d)^{\alpha}$, and $\tau_{3}^{\alpha}$ by

$$
\left(\tau_{3}^{\alpha \lambda} \omega\right)\left(X_{1}, Y_{\alpha}, Z_{\alpha}\right)=\sum_{k-1}^{\alpha} \omega\left(X_{1},(h-\lambda i d)^{\alpha-k} Y_{\alpha},(h-\lambda i d)^{k-1} Z_{\alpha}\right)
$$

$\forall X_{1} \in E_{1}, \forall Y_{\alpha}, Z_{\alpha} \in \operatorname{Ker}(h-\lambda i d)^{\alpha}$. However the results which involve the vanishing of Nijenhuis tensor - in particular Proposition 1.1 and the properties which can be deduced from it, such as 2.2 etc. - cannot be generalized in an obvious way because the Nijenhuis torsion of $g:=h-\lambda i d$ does not vanish in general. Indeed, $\frac{1}{2}[g, g]=\frac{1}{2}[h, h]-d_{h} \lambda \wedge i d+d \lambda \wedge h$. Now from the identities $[h X, h]=h[X, h]$ and $[h, X] h+h[h, X]=\left[h^{2}, X\right]$, which hold for any $X \in \underline{T}$ and for any $h$ such that $[h, h]=0$, one can deduce that $d_{h}(\operatorname{Tr} h)=\frac{1}{2} d \operatorname{Tr}^{2}$ and, in our case, $d_{h} \lambda=\lambda d \lambda$. Thus

$$
\frac{1}{2}[g, g]=d \lambda \wedge g
$$

In particular, if $\lambda$ is a constant (this is the so-called "0-deformable" case) one has $\frac{1}{2}[g, g]=0$, and then $d d_{h}=d d_{g}$. Therefore all the results of preceding sections can be applied immediately and, in particular, one has:

Let $h$ be 0 -deformable with minimal polynomial $(X-\lambda)^{p}, p \geq 2$. If $\operatorname{Ker}(h-\lambda i d)$, $\operatorname{Ker}(h-\lambda i d)^{2}, \cdots, \operatorname{Ker}(h-\lambda i d)^{p-1}$ are involutive, then $d d_{h}$ is formally integrable.

In order to generalize the result to the case where the eigenvalue is not constant we need the following lemma.

Lemma 3.1. 1. Let $h \in \underline{T^{*} \otimes T}$ and $\lambda \in \mathcal{C}^{\infty}(M)$ and denote by $\rho_{\alpha}^{\lambda}: \wedge^{2} T^{*} \longrightarrow$ $\wedge^{2} T^{*}$ the map defined by $\overline{\left(\rho_{\alpha}^{\lambda} \omega\right)}(X, Y)=\sum_{k=1}^{\alpha} \omega\left(g^{\alpha-k} X, g^{k-1} Y\right)$, where $g:=$ $h-\lambda i d$. Set $g^{\alpha}=\sum_{k=0}^{\alpha} b_{k}^{\alpha} h^{\alpha}$, where $b_{k}^{\alpha}:=\left(\begin{array}{c}\alpha \\ k\end{array}\right)(-\lambda)^{\alpha-k} ;$ then

$$
\rho_{\alpha}^{\lambda}=\sum_{k=1}^{\alpha} b_{k}^{\alpha} \rho_{k} .
$$

2. If $\widetilde{\rho}_{\alpha}^{\lambda}: \wedge^{2} T^{*} \longrightarrow \wedge^{2}\left(\operatorname{Ker} g^{\alpha}\right)^{*}$ is the map defined by $\widetilde{\rho}_{\alpha}^{\lambda}=\left.\rho_{\alpha}^{\lambda}\right|_{\wedge^{2}\left(\operatorname{Kerg} g^{\alpha}\right)}$, then

$$
\widetilde{\rho}_{\alpha}^{\lambda} d d_{h} f=-C_{g^{\alpha}}+\left.\alpha d \lambda \wedge g^{\alpha-1}\right|_{\wedge^{2}\left(\operatorname{Kerg}^{p-1}\right)^{*}} \quad(\alpha=1, \cdots, p),
$$

where $C_{g^{\alpha}}$ is defined in 2.1.

Proof. 1. We have

$$
\begin{gathered}
\left(\rho_{\alpha}^{\lambda} \omega\right)(X, Y)=\sum_{k=1}^{\alpha} \omega\left(\sum_{r=0}^{\alpha-k}\left(\begin{array}{c}
\alpha-k \\
r
\end{array}\right)(-\lambda)^{\alpha-k-r} h^{r} X, \sum_{s=0}^{k-1}\left(\begin{array}{c}
k-1 \\
s
\end{array}\right)(-\lambda)^{k-1-s} h^{s} Y\right) \\
=\sum_{k=1}^{\alpha} \sum_{s=0}^{k-1} \sum_{r=0}^{\alpha-k}(-\lambda)^{\alpha-1-r-s}\left(\begin{array}{c}
\alpha-k \\
r
\end{array}\right)\left(\begin{array}{c}
k-1 \\
s
\end{array}\right) \omega\left(h^{r} X, h^{s} Y\right) .
\end{gathered}
$$


If we put $t:=r+s$, we obtain

$(*)$

$$
\begin{aligned}
\left(\rho_{\alpha}^{\lambda} \omega\right)(X, Y) & =\sum_{k=1}^{\alpha} \sum_{s=0}^{k-1} \sum_{t=s}^{\alpha-k+s}\left(\begin{array}{c}
\alpha-k \\
t-s
\end{array}\right)\left(\begin{array}{c}
k-1 \\
s
\end{array}\right) \omega\left(h^{t-s} X, h^{s} Y\right)(-\lambda)^{\alpha-1-t} \\
& =\sum_{t=0}^{\alpha-1} \sum_{s=0}^{t} \sum_{k=s+1}^{\alpha+s-t}\left(\begin{array}{c}
\alpha-k \\
t-s
\end{array}\right)\left(\begin{array}{c}
k-1 \\
s
\end{array}\right)(-\lambda)^{\alpha-1-t} \omega\left(h^{t-s} X, h^{s} Y\right) \\
& =\sum_{t=0}^{\alpha-1} \sum_{s=0}^{t}\left(\begin{array}{c}
\alpha \\
t+1
\end{array}\right)(-\lambda)^{\alpha-1-t} \omega\left(h^{t-s} X, h^{s} Y\right) \\
& =\sum_{k=1}^{\alpha} \sum_{r=1}^{k}\left(\begin{array}{c}
\alpha \\
k
\end{array}\right)(-\lambda)^{\alpha-k} \omega\left(h^{k-r} X, h^{r-1} Y\right)=\sum_{k=1}^{\alpha}\left(b_{k}^{\alpha} \rho_{k} \omega\right)(X, Y) .
\end{aligned}
$$

2. From 1 it follows that

$$
\begin{gathered}
\rho_{\alpha}^{\lambda} d d_{h} f=\sum_{k=1}^{\alpha} b_{k}^{\alpha} \rho_{k} d d_{h} f=\sum_{k=1}^{\alpha} b_{k}^{\alpha} d d_{h^{k}} f=\sum_{k=1}^{\alpha}\left(d\left(b_{k}^{\alpha} d_{h^{k}} f\right)-\left(d b_{k}^{\alpha} \wedge d f\right)\right. \\
=d d_{g^{\alpha}} f-\sum_{k=1}^{\alpha} d f \circ\left(d b_{k}^{\alpha} \wedge h^{k}\right)=d d_{g^{\alpha}} f+d f \circ\left(\alpha d \lambda \wedge g^{\alpha-1}\right)
\end{gathered}
$$

and then $\quad \widetilde{\rho}_{\alpha}^{\lambda} d d_{h} f=d f \circ\left(-C_{g^{\alpha}}+\alpha d \lambda \wedge g^{\alpha-1}\right)$.

Let us set

$$
C_{\alpha}^{\lambda}:=-C_{g^{\alpha}}+\left.\alpha d \lambda \wedge g^{\alpha-1}\right|_{\wedge^{2}\left(\operatorname{Ker} g^{\alpha}\right)^{*}}
$$

and let $\mathbb{K}_{\alpha}^{\lambda}$ be the subspace of $\operatorname{Im} g^{\alpha-1} \cup \operatorname{Ker} g^{\alpha}$ spanned by the image of $C_{\alpha}^{\lambda}$. The following proposition can be proved in the same way as Proposition 2.2.

Proposition 3.1. Suppose that $[h, h]=0$ and that the minimal polynomial of $h$ is $(X-\lambda)^{p}, p \geq 2$. If $R_{2}$ denotes the space of second order formal solutions of the operator $d d_{h}$ at $x_{o} \in M$ and $\bar{\pi}_{r}:\left(R_{2}\right) \longrightarrow T_{x_{o}}^{*}$ is the restriction $\bar{\pi}_{2}=\pi_{\left.\right|_{2}}$, then

$$
\bar{\pi}_{2}\left(R_{2}\right)=\left(\mathbb{K}_{1}^{\lambda}\right)^{o} \cap \cdots \cap\left(\mathbb{K}_{p}^{\lambda}\right)^{o}
$$

The following corollary can be deduced in the same way as Proposition 2.1 and Proposition 2.2 :

Corollary 3.1. 1. Under the hypotheses of Proposition 3.1, a form $\omega \in T_{x_{o}}^{*}$ can be lifted to a second order solution if and only if $\omega \circ C_{\alpha}^{\lambda}=0$ for any $\alpha=1, \ldots, p-1$. Each $\omega \in T_{x_{o}}^{*}$ such that $h^{*} \omega=\lambda \omega$ can be lifted to a second order formal solution at $x_{o}$. In particular, $\bar{\pi}_{2}\left(R_{2}\right) \neq(0)$.

2. Every form in $T^{*}$ can be lifted to a second order formal solution if and only if $C_{\alpha}^{\lambda}=0$ for any $\alpha=1, \ldots, p$.

In order to check the formal integrability of $d d_{h}$, we only have to prove that for any linear connection $\nabla$ on $M$ one has $\tau_{3}^{\alpha, \lambda}\left(\nabla d d_{h} f\right)_{x_{o}}=0$ if $\left(d d_{h} f\right)_{x_{o}}=0$. Now 
for each $X \in E_{1}$ and $Y_{\alpha}, Z_{\alpha} \in \operatorname{Ker}(h-\lambda i d)^{\alpha}$,

$$
\begin{aligned}
\tau_{3}^{\alpha, \lambda} & \left(\nabla d d_{h} f\right)_{x_{o}}\left(X, Y_{\alpha}, Z_{\alpha}\right)=\sum_{\operatorname{cycl}(\mathrm{X}, \mathrm{Y}, \mathrm{Z})} \nabla_{X}\left(\rho_{\alpha}^{\lambda} d d_{h} f\right)\left(Y_{\alpha}, Z_{\alpha}\right)_{x_{o}} \\
& =\sum_{k=1}^{\alpha}\left(\nabla_{X} d d_{h} f\right)\left((h-\lambda i d)^{d-k} Y_{\alpha},(h-\lambda i d)^{k-1} Z_{\alpha}\right)_{x_{o}} \\
& =\nabla_{X}\left(\left(\widetilde{\rho}_{\alpha} d d_{h} f\right)\left(Y_{\alpha}, Z_{\alpha}\right)=-\nabla_{X}\left(d f \circ C_{\alpha}^{\lambda}\right)\left(Y_{\alpha}, Z_{\alpha}\right)\right.
\end{aligned}
$$

Then, if as in section 2.2 we denote by $C_{\alpha}^{\lambda^{*}}$ the transpose of $C_{\alpha}^{\lambda}$ defined by $\left(C_{\alpha}^{\lambda^{*}}(\omega)\right)(U, V):=\omega\left(C_{\alpha}^{\lambda}(U, V)\right)$, then under the hypotheses of Proposition 3.1 we have the following theorem:

Theorem 3.1. The differential operator $d d_{h}$ is formally integrable at $x_{o}$ if and only if, for any second order solution $F_{o}$ at $x_{o}$,

$$
\left(p_{1} C_{\alpha}^{\lambda^{*}}\right)\left(F_{o}\right)=0 \quad(\alpha=1, \ldots, p)
$$

where $p_{1} C_{\alpha}^{\lambda^{*}}$ denotes the first prolongation of $C_{\alpha}^{*}$ (here we have identified $T_{x_{o}}^{*}$ with $\left.J_{1, x_{o}} \mathbb{R} / J_{o, x_{o}} \mathbb{R}\right)$.

\section{Geometrical interpretation of the obstruction.}

Definition 3.1. Let $h$ be an endomorphism field of $T M$ and $\lambda$ an eigenvalue of $h$ whose multiplicity in the minimal polynomial is $p$. We say that the characteristic flag associated to $\lambda$ is integrable if the distributions $\operatorname{Ker}(h-\lambda i d)$, $\operatorname{Ker}(h-\lambda i d)^{2}, \cdots, \operatorname{Ker}(h-\lambda i d)^{p}$ are involutive. The leaves of the integrable distribution $\operatorname{Ker}(h-\lambda i d)^{p}$ (respectively, $\left.\operatorname{Ker}(h-\lambda i d)^{p-1}\right)$, are called "maximal leaves" of the characteristic flag (respectively : "maximal proper leaves" of the characteristic flag).

Proposition 3.2. The following two statements are equivalent:

1. $C_{\alpha}^{\lambda}=0$ for any $\alpha=1, \cdots, p$.

2. The characteristic flag is involutive and $d \lambda \wedge g^{p-1}=0$. This last condition is equivalent to the following: either $\lambda$ is constant, or the codimension of the maximal proper leaves is 1 and $\lambda$ remains constant on these leaves.

Indeed, if $\lambda$ is constant, $C_{\alpha}^{\lambda}=0$ for any $\alpha=1, \cdots, p$ is equivalent to $C_{g^{\alpha}}=0$, which means that the characteristic flag is involutive.

Suppose that $d \lambda \neq 0$. For $\alpha=p$ the condition 1 is $d \lambda \wedge g^{p-1}=0$, which means that $\operatorname{Ker} d \lambda=\operatorname{Ker} g^{p-1}$ (in particular, $\lambda$ is constant on the leaves and the rank of $g^{p-1}$ is 1). The condition $C_{p-1}^{\lambda}=0$ gives

$$
-C_{g^{p-1}}+\left.(p-1) d \lambda \wedge g^{p-2}\right|_{\wedge^{2}\left(\operatorname{Ker} g^{p-1}\right)}=0
$$

that is, $C_{g^{p-1}}=0$, because $\operatorname{Ker} d \lambda=\operatorname{Ker} g^{p-1}$. By induction one can easily see that $C_{\alpha}^{\lambda}=0$ for all $\alpha=1, \cdots, p$ is equivalent to the integrability of the characteristic flag.

Finally, we have

Theorem 3.2. Let $h \in \underline{T^{*} \otimes T}$ be an endomorphism field with $[h, h]=0$, and suppose that the minimal polynomial of $h$ is $(X-\lambda)^{p}(p>1)$. If the characteristic flag is integrable and $d \lambda \wedge(h-\lambda i d)^{p-1}=0$ (that is, either $\lambda$ is constant, or the codimension of the maximal proper leaves is 1 and $\lambda$ remains constant on these leaves), then $d d_{h}$ is formally integrable. 
Theorem 3.3. (Normal form of $h$ ). Let $h$ be an analytic endomorphism with $[h, h]=0$, and suppose that its minimal polynomial is $(X-\lambda)^{p}(p \geq 2)$. Fix a point $x_{o} \in M$. The following two statements are equivalent

1. There exists a neighborhood $U$ of $x_{o}$ such that every $x \in U$ admits a complete system of conservation laws (i.e. every $\omega_{o} \in T_{x}^{*}$ can be prolonged in a germ of conservation laws).

2. The characteristic flag is involutive and $d \lambda \wedge g^{p-1}=0$ (i.e., either $\lambda$ is constant, or the codimension of the maximal proper leaves is 1 and $\lambda$ remains constant on these leaves).

If the above statements hold, then for suitable local coordinates in a neighborhood of $x_{o}, h$ takes the form

$$
H=\left(\begin{array}{cccc}
J_{q_{1}} & & & \\
& J_{q_{2}} & & \\
& & \ddots & \\
& & & J_{q_{s}}
\end{array}\right),
$$

where $q_{1}, q_{2}, \cdots, q_{s}$ are the degrees of the elementary divisors,

$$
J_{q_{r}}=\left(\begin{array}{llll}
0 & 1 & & 0 \\
\vdots & \ddots & \ddots & \\
0 & \cdots \cdots & 0 & 1 \\
c_{q_{r}} & \cdots \cdots & c_{2} & c_{1}
\end{array}\right)
$$

is the block corresponding to the elementary divisor $(X-\lambda)^{q_{j}}$, and

$$
c_{k}=(-1)^{k+1}\left(\begin{array}{c}
q_{r} \\
k
\end{array}\right) \lambda^{k}
$$

(If $p=1$, condition 1 is satisfied if and only if the dimension of $M$ is 1.)

3.2. The general case. We suppose now that the minimal polynomial of $h$ is

$$
m_{h}(X)=\left(X-\lambda_{1}\right)^{p_{1}} \cdots\left(X-\lambda_{r}\right)^{p_{r}} .
$$

Taking into account the identity $\left[g^{p_{i}}, g^{p_{i}}\right]=\sum_{k=0}^{2 p_{i}-1} a_{k} d_{g^{k}} \lambda \wedge g^{2 p_{i}-k-1}$, where $a_{k} \in \mathbb{R}$ are appropriate constants, it is not difficult to prove the following property:

Lemma 3.2. The characteristic subspaces $G_{i}:=\operatorname{Ker}\left(h-\lambda_{i} i d\right)^{p_{i}}(i=1, \cdots, r)$ are involutive and $\left[h_{i}, h_{i}\right]=0$, where $h_{i}:=h_{\left.\right|_{i}}$.

Our main theorem can now be proved by restricting to the characteristic subspaces. Namely, let us suppose first that in the minimal polynomial $p_{i} \geq 2$ for every $i=1, \ldots, r$ and that condition 2 in Theorem 3.3 is satisfied for every $G_{i}$ and for every $h_{i}$. Let $\omega_{o} \in T_{x_{o}}^{*}$ and consider its decomposition on the dual spaces of $G_{i}$ : $\omega_{o}=\alpha_{1}+\cdots+\alpha_{r}$. There exist functions $f_{i}$ defined on neighborhoods $U_{i}$ of $x_{o}$ (in the maximal submanifold of $G_{i}$ through $\left.x_{o}\right)$ such that $\alpha_{i}=\left(d f_{i}\right)_{x_{o}}$ and $d d_{h_{i}} f_{i}=0$. Set $U=U_{1} \times \cdots \times U_{r}$ and let $\widetilde{f}_{i}: U \longrightarrow \mathbb{R}$ be the map defined by $\widetilde{f}_{i} \mid U_{j}=\delta_{i j} f_{j}$. If $f:=\sum_{i=1}^{r} \tilde{f}_{i}$ one has clearly $\omega_{o}=(d f)_{x_{o}}$ and $d d_{h} f=0$. Then $\omega_{o}$ can be prolonged to a conservation law.

Conversely, suppose that there exists a neighborhood $U$ of $x_{o}$ such that, for any $y \in U$, every $\omega \in T_{y}^{*}$ can be prolonged to a conservation law. Let us decompose $U=$ $U_{1} \times \cdots \times U_{r}$ as above, and write $y=\left(y_{1}, \ldots, y_{r}\right)$. Considering a form $\alpha_{1} \in T_{y_{1}}^{*} U_{1}$ as a form of $T_{y}^{*} U$, we can construct a conservation law $d f$ on $U$ such that $(d f)_{y}=\alpha_{1}$. It 
is not difficult to see that the function $f_{1}$ on $U_{1}$ defined by $f_{1}\left(x_{1}\right)=f\left(x_{1}, y_{2}, \ldots, y_{r}\right)$ gives rise to a conservation law $d f_{1}$ on $U_{1}$ such that $\left(d f_{1}\right)_{y_{1}}=\alpha_{1}$ (this is due to the fact that $G_{i}$ is integrable: consider, for example, the expression of $d d_{h} f$ in local coordinates adapted to the decomposition $\left.U=U_{1} \times \cdots \times U_{r}\right)$. Then $h_{i}$ satisfies condition 2 of Theorem 3.3.

Therefore, taking into account the remark in footnote 1 of section 3.1 for the case where an eigenvalue has multiplicity 1 in the minimal polynomial, and noting that the vector space spanned by the image of $C_{s}^{\lambda_{i}}$ is included in $G_{i}$ for any $s$, we can state:

Theorem 3.4. Let $h$ be an analytic endomorphism field with $[h, h]=0$, and suppose that $h$ has a constant "algebraic type" with minimal polynomial

$$
m_{h}(X)=\left(X-\lambda_{1}\right)^{p_{1}} \cdots\left(X-\lambda_{r}\right)^{p_{r}} .
$$

Then:

1. A 1-form $\omega_{o} \in T_{x_{o}}^{*} \simeq\left(J_{1}\right)_{x_{o}} \mathbb{R}$ can be lifted to a second order solution $F_{o}$ of the differential operator $d d_{h}$ if and only if

$$
C_{s}^{\lambda_{i}{ }^{*}}\left(\omega_{o}\right)=0
$$

for every eigenvalue $\lambda_{i}$ and for every $s=1, \ldots, p_{i}$.

2. The differential operator $d d_{h}$ is formally integrable at $x_{o}$ if and only if for every eigenvalue $\lambda_{i}$ and for every $s=1, \ldots, p_{i}$ one has

$$
\left(p_{1} C_{s}^{\lambda_{i}{ }^{*}}\right)\left(F_{o}\right)=0
$$

for every second-order solution $F_{o}$, where $p_{1} C_{s}^{\lambda_{i}{ }^{*}}$ is the first prolongation of $C_{s}^{\lambda^{*}}$.

3. In particular, the following two statements are equivalent:

(a) There exists a neighborhood $U$ of $x_{o}$ such that every $x$ admits a complete system of germs of conservation laws (i.e. every $\omega_{o} \in T_{x}^{*}$ can be extended to a germ of conservation laws).

(b) Let $\lambda$ be an eigenvalue of $h$ with multiplicity $p$ in the minimal polynomial. If $p=1$, then the maximal leaves of his characteristic flag are 1-dimensional; if $p \geq 2$ then either $\lambda$ is constant on the maximal leaves, or $\lambda$ is constant on the maximal proper leaves, which necessarily are 1codimensional in the maximal leaves.

In this case there exist local coordinates in a neighborhood of $x_{o}$ such that $h$ has the "normal form"

$$
\left(\begin{array}{ccc}
H_{1} & & 0 \\
& \ddots & \\
0 & & H_{r}
\end{array}\right),
$$

$H_{i}$ being the same blocks as in Theorem 3.3.

Remark. The above theorem remains true in the case when $h$ has complex eigenvalues, and the proofs are the same. Indeed if $\omega_{x_{o}}$ is a real 1 -form, the real part of the complex conservation law whose existence is ensured by Theorem 3.4 will be the real conservation law which takes the value $\omega_{x_{o}}$ at $x_{o}$. 


\section{Appendix 1. Proof of Lemma 2.2}

We shall use the technique of successive separations of equations, which is explained in [2]. For more details cf. [12].

The demonstration can be divided into three steps, according to the number of cyclic subspaces which arise.

STEP 1. Each block $B_{i i i}^{\theta}$ contains only independent equations. The statement is proved in [2], because, for each $i$ the subspace $V_{i}$ is cyclic.

STEP 2. Each block $B_{j i i}^{\theta}$, with $i \neq j$, contains only independent equations. Suppose that $i<j$ (the proof in the case $i \geq j$ is similar: for more details cf. [12]).

a) Case $\theta \leq 1+q_{i}+q_{j}$.

Lemma 1. Let $\bar{\alpha}$ be fixed and denote by $B_{1}^{\bar{\alpha}}$ the subblock $B_{j i i}^{\theta}$ consisting of the equations $L_{j i}^{\bar{\alpha} \beta \gamma}$, by $B_{2}^{\bar{\alpha}}$ the subblock consisting of the equations $M_{j i i}^{\bar{\alpha} \beta \gamma}$, and $B^{\bar{\alpha}}=$ $B_{1}^{\bar{\alpha}} \cup B_{2}^{\bar{\alpha}}$. The blocks $B^{\bar{\alpha}}$ are independent and contain only independent equations.

\section{SCHEMA 1}

\begin{tabular}{|c|c|c|c|}
\hline pivots & $B_{1}^{\bar{\alpha}}$ & $B_{2}^{\bar{\alpha}}$ & property satisfied for \\
\hline$C_{i_{i} \gamma_{i}}^{1 \beta \gamma}$ & $\frac{1 \beta \gamma}{L_{j i}^{1 \beta \gamma}}$ & & \\
\hline$C_{i}^{\bar{\alpha}+11 i \gamma}$ & $\left(L_{j \underset{i}{1} i_{i}^{\gamma+1}}^{\bar{\alpha}+1}\right)$ & $M_{j i i}^{1 \gamma \bar{\alpha}}$ & $B^{1}$ \\
\hline$\ldots$ & ...(induction on $\bar{\alpha}) \ldots$ & & \\
\hline$C_{j}^{\bar{\alpha}+1}{ }_{i}^{\beta \gamma}{ }_{i} \gamma$ & $L_{j}^{\bar{\alpha}+1}{ }_{i}^{\beta \gamma} \gamma$ & $\left(M_{j i i}^{\bar{\alpha} \beta \gamma}\right)$ & $B^{\bar{\alpha}}$ \\
\hline$C_{i}^{\gamma+1} \underset{j}{\bar{\alpha}+1} \beta$ & $\left(L_{j}^{\bar{\alpha}+1}{ }_{i}^{\beta}{ }_{i}^{\gamma+1}\right)$ & $M_{j}^{\bar{\alpha}+1}{ }_{i}^{\beta}{ }_{i}$ & $B^{\bar{\alpha}+1}$ \\
\hline
\end{tabular}

The proof follows Schema 1, which summarizes the following induction argument. For every $\beta$ and $\gamma, C_{j i i}^{1 \beta \gamma}$ is a pivot for equation $L_{j i i}^{1 \beta \gamma}$ : therefore these equations are separable. On the other hand $C_{j i i}^{\bar{\alpha}+1 \beta \gamma}$ appears only in $L_{j i i}^{1 \gamma \bar{\alpha}+1}$ (this equation has just been separated) and in $M_{j i i}^{1 \gamma \bar{\alpha}}$ (which becomes separable). Therefore the block $B^{1}$ is separable and contains only independent equations. Suppose that the property is satisfied at order $\bar{\alpha} . C_{j}^{\bar{\alpha}+1 \beta \gamma}{ }_{i i}$ appears only in $L_{j i i}^{\bar{\alpha}+1 \beta \gamma}$ and in $M_{j i i}^{\bar{\alpha} \beta \gamma}$, which has been separated by the induction hypothesis. Then $L_{j i i}^{\bar{\alpha}+1}{ }^{\beta} \gamma$ is separable. Finally, $C_{i j i}^{\gamma+1 \bar{\alpha}+1 \beta}$ appears only in $L_{j i i}^{\bar{\alpha}+1 \beta \gamma+1}$, which becomes separable. Thus the property is satisfied for the block $B^{\bar{\alpha}+1}$.

Now :

$\diamond \quad$ If $\theta<2+q_{i}$, then the blocks $B_{j i i}^{\theta}$ do not involve equations arising from $\tau_{3}^{\alpha}$ (i.e. $\left.N_{i i j}^{1 u v}\right)$. Therefore the property follows from Lemma 1.

$\diamond \quad$ If $2+q_{i} \leq 1+q_{i}+q_{j}$, then the block $B_{j i i}^{\theta}$ contains the equations of the lemma and additional equations of the type $N_{i i j}^{1 \theta-q_{j}-1 \theta-q_{i}-1}$. The proof is carried out as in Lemma 1,because the pivots chosen do not appear in these equations, and these equations are independent, as is easily proved.

b) Case $\theta>1+q_{i}+q_{j}$.

In this case, $B_{j i i}^{\theta}$ does not contain any equations arising from $\tau_{3}^{\alpha}$. Let us fix $\bar{\alpha}$, and denote by $B_{1}^{\bar{\alpha}}$ the set of equations $L_{j}^{q_{i}-\bar{\alpha}+1 \underset{i}{\beta} \gamma}$, by $B_{2}^{\bar{\alpha}}$ the set of equations 
$M_{j}^{q_{i}-\bar{\alpha}+1 \beta \gamma}{ }_{i i}$, and set $B^{\bar{\alpha}}=B_{1}^{\bar{\alpha}} \cup B_{2}^{\bar{\alpha}}$. Clearly the subblocks $B_{1}^{\bar{\alpha}}$ and $B_{2}^{\bar{\alpha}} \operatorname{cover} B_{j i i}^{\theta}$; we shall prove, by induction, that they are successively separable.

$\diamond B^{1}$ is separable. Set $\theta=r+q_{i}+q_{j} \quad(r>1)$. The argument is slightly different depending on whether $r+q_{i}$ is even or odd.

For $r+q_{i}=2 p+1$ the proof follows Schema 2 (which should be read in the same way as Schema 1):

SCHEMA 2

\begin{tabular}{|c|c|c|c|}
\hline pivots & $B_{1}^{1}$ & $B_{2}^{1}$ & property satisfied for \\
\hline $\begin{array}{l}C_{i j i}^{p+1} q_{j} p \\
C^{p} q_{j} p+1\end{array}$ & $\begin{array}{l}L_{j i}^{q_{j}} p_{i} p+1 \\
q_{j} p-1 \quad p+2\end{array}$ & & \\
\hline$C_{i j i}^{p q_{j}{ }^{p+1}}$ & $\begin{array}{l}\left(L_{j i}^{q_{j}{ }^{p-1} i}{ }^{p+2}\right) \\
\ldots(\text { induction on } s)\end{array}$ & $M_{j i}^{q_{j}{ }^{p-1}{ }^{p+1}} i$ & $B^{1}$ \\
\hline$C_{i}^{p-s \underset{j}{q_{j}} \underset{i}{p+s+1}}$ & $\left(\begin{array}{ccc}L_{j}^{q_{j}} & p-s & p-s+1 \\
i & i\end{array}\right)$ & $M_{j}^{q_{j}} \underset{i}{p-s-1} p-s+1$ & $B^{s}$ \\
\hline$C_{i}^{p+s+2} \underset{j}{q_{j}}{ }_{i}^{p-s-1}$ & $L_{j i}^{q_{j}{ }^{p-s-1}{ }_{i}^{p-s+2}}$ & $\left(M_{j i}^{q_{j}}{ }_{i}^{p-s-1}{ }^{p-s+1}\right)$ & $B^{s+1}$ \\
\hline
\end{tabular}

For $r+q_{i}=2 p$ one follows Schema 3:

SCHEMA 3

\begin{tabular}{|c|c|c|c|}
\hline pivots & $B_{1}^{1}$ & $B_{2}^{1}$ & property satisfied for \\
\hline$C_{i{ }_{i j}{ }_{j} q_{j}}^{p q}$ & & $M_{j i i}^{q_{j} p-1 p}$ & \\
\hline$C_{i}^{p+1}{ }_{j i}^{q_{j}}{ }^{p-1}$ & $L_{j i}^{q_{j}{ }^{p-1}{ }_{i}^{p+1}}$ & $\left(M_{j i}^{q_{j}}{ }^{p-1}{ }_{i}^{p}\right)$ & $B^{1}$ \\
\hline$\ldots$ & ...(induction on $s$ ) & $\cdots$ & \\
\hline$C_{i}^{p-s} \underset{i}{p+s} q_{j}$ & 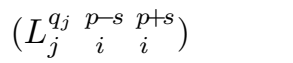 & $M_{j}^{q_{j}} \underset{i}{p-s-1}{ }_{i}^{p+s}$ & $B^{s}$ \\
\hline$C_{i}^{p-s-1} \underset{j}{q_{j}} q_{j}^{p+s+1}$ & $L_{j}^{q_{j}} \underset{i}{p-s-1} \underset{i}{p+s+1}$ & $\left(\begin{array}{ccc}M_{j}^{q_{j}} & p-s-1 & p+s \\
i\end{array}\right)$ & $B^{s+1}$ \\
\hline
\end{tabular}

$\diamond$ Suppose now that $B^{1}, B^{2}, \ldots, B^{\bar{\alpha}-1}$ are successively separable; we prove that $B^{\bar{\alpha}}$ is separable.

Let us consider $M_{j}^{q_{j}-\bar{\alpha}+1{ }_{i}{ }_{i}^{\gamma}} \in B_{2}^{\bar{\alpha}}$. This equation is separable by the pivot $C_{j}^{q_{j}-\bar{\alpha}+2 \beta \gamma}{ }_{i i}$, which appears only in this equation and in $L_{j}^{q_{j}-\bar{\alpha}+2{ }_{i i} \gamma}$ (which lies in $B_{1}^{\bar{\alpha}-1}$, and is already separated by induction hypothesis). Therefore $B_{2}^{\bar{\alpha}}$ is separable. In the same way $L_{j}^{q_{j}-\bar{\alpha}+1 \beta \gamma}{ }_{i}{ }_{i}^{\gamma} \in B_{1}^{\bar{\alpha}}$ is separable because $C_{i j}^{\gamma}{ }_{j}^{q_{j}-\bar{\alpha}+1}{ }_{i}^{\beta}$ appears only in this equation and in $B_{2}^{\bar{\alpha}}$. This completes the proof in case 2 .

STEP 3. Every block $B_{i j k}^{\theta}$ with $i<j<k$ is formed by independent equations if $\theta<2+q_{i}$ and by equations related by only one linear relation if $\theta \geq 2+q_{i}$. Note that, since $\theta \leq 1+q_{i}+q_{j}+q_{k}$, this statement completes the proof of Lemma 2.2. Suppose that $2+q_{i} \leq 1+q_{j}+q_{k}$ (if $2+q_{i}>1+q_{j}+q_{k}$, the proof is similar).

Lemma 2. Fix $\bar{\alpha}$, and let $B_{1}^{\bar{\alpha}_{1}}$ denote the subblock formed by the equations of type $L_{k j i}^{\bar{\alpha} \beta \gamma}, B_{2}^{\bar{\alpha}}$ the subblock of equations $M_{k j i}^{\bar{\alpha} \beta \gamma}$, and set $B^{\bar{\alpha}}=B_{1}^{\bar{\alpha}} \cup B_{2}^{\bar{\alpha}}$. If $\theta \leq 1+q_{i}+q_{j}$, the blocks $B^{\bar{\alpha}}$ are independent and contain only independent equations.

The proof follows Schema 4: 


\section{SCHEMA 4}

\begin{tabular}{||l|ll|c||}
\hline pivots & $B_{1}^{\bar{\alpha}}$ & $B_{2}^{\bar{\alpha}}$ & property satisfied for \\
\hline$C_{k j i}^{1 \beta \gamma}$ & $L_{k j i}^{1 \beta \gamma}$ & & \\
$C_{i k j}^{\gamma+1} \operatorname{li}_{k} \beta$ & $\left(L_{k j i}^{1 \beta \gamma+1}\right)$ & $M_{k j i}^{1 \beta \gamma}$ & $B^{1}$ \\
$\ldots$ & $\ldots($ induction on $\bar{\alpha}) \ldots$ & & \\
$C_{k}^{\bar{\alpha}+1 \beta \gamma}$ & $L_{k}^{\bar{\alpha}+1 \beta \gamma}$ & $\left(M_{k j i}^{\bar{\alpha} \beta \gamma}\right)$ & $B^{\bar{\alpha}}$ \\
$C_{i k j}^{\gamma+1 \bar{\alpha}+1 \beta}$ & $\left(L_{k j i}^{\bar{\alpha}+1 \beta \gamma}\right)$ & $M_{k j i}^{\bar{\alpha}+1 \beta \gamma}$ & $B^{\bar{\alpha}+1}$ \\
\hline
\end{tabular}

a) Case $\theta<2+q_{i}$. We have to prove that the equations of $B_{i j k}^{\theta}$ are independent.

$\diamond \quad$ If $\theta<2+q_{j}, B_{i j k}^{\theta}$ contains only the blocks $B^{\bar{\alpha}}$ of Lemma 2 and in this case the property follows from Lemma 2 .

$\diamond$ If $2+q_{j} \leq \theta<2+q_{i}, B_{i j k}^{\theta}$ contains not only the blocks $B^{\bar{\alpha}}$ of the lemma, but equations of the type $N_{i}^{1}{ }_{j}^{\theta-q_{k}+1}{ }_{j}^{\theta-q_{j}+1}$ too. Now these equations are independent and do not contain the pivots used in the proof of the lemma. Thus the property is true in this case.

b) Case $\theta \geq 2+q_{i}$. We have to prove that the equations of $B_{i j k}^{\theta}$ are related by only one linear relation. Note that

$$
2+q_{i} \leq 1+q_{j}+q_{k} \leq 1+q_{i}+q_{k} \leq 1+q_{i}+q_{j} .
$$

We have to consider four cases. We give here only the results; for details the reader may see $[12]^{2}$

$\diamond$ i) $2+q_{i} \leq \theta \leq 1+q_{j}+q_{k}$. In this case $B_{i j k}^{\theta}$ contains the blocks $B^{\bar{\alpha}}$ of the lemma and the equations $N_{i j k}^{1 w v}, \quad N_{j i k}^{1 w u}, \quad N_{k i j}^{1 v u}$, where : $u=\theta-1-q_{i}, v=$ $\theta-1-q_{j}, w=\theta-1-q_{k}$. One has

1. $\sum_{\alpha+\beta+\gamma=\theta} L_{k j i}^{\alpha \beta \gamma}=\sum_{\alpha+\beta+\gamma=\theta-1} M_{k j i}^{\alpha \beta \gamma}-N_{i j k}^{1 w v}+N_{j i k}^{1 w u}-N_{k i j}^{1 v u}$.

2. The equations of $B_{i j k}^{\theta} \backslash\left\{N_{k i j}^{1 v u}\right\}^{3}$ are independent.

$\diamond$ ii) $1+q_{j}+q_{k}<\theta \leq 1+q_{i}+q_{k}$. In this case $B_{i j k}^{\theta}$ contains the blocks $B^{\bar{\alpha}}$ and the equations $N_{j i k}^{1 w u}$ and $N_{k i j}^{1 v u}$. One has

1. $\sum_{\alpha+\beta+\gamma=\theta} L_{i j k}^{\alpha \beta \gamma}=\sum_{\alpha+\beta+\gamma=\theta-1} M_{i j k}^{\alpha \beta \gamma}+N_{j i k}^{1 w u}-N_{k i j}^{1 v u}$.

2. The equations of $B_{i j k}^{\theta} \backslash\left\{N_{k i j}^{1, v, u}\right\}$ are independent.

$\diamond$ iii) $1+q_{i}+q_{k}<\theta \leq 1+q_{i}+q_{j}$. In this case $B_{i j k}^{\theta}$ contains only the blocks $B^{\bar{\alpha}}$ and the equation $N_{k i j}^{1 v u}$. One has:

1. $\sum_{\alpha+\beta+\gamma=\theta} L_{i j k}^{\alpha \beta \gamma}=\sum_{\alpha+\beta+\gamma=\theta-1} M_{i j k}^{\alpha \beta \gamma}-N_{k i j}^{1 u u}$.

2. The equations of $B_{i j k}^{\theta} \backslash\left\{N_{k i j}^{1 v u}\right\}$ are independent.

$\diamond$ iv) $\theta>1+q_{i}+q_{j}$. In this case $B_{i j k}^{\theta}$ contains only the blocks $B^{\bar{\alpha}}$.

1. $\sum_{\alpha+\beta+\gamma=\theta} L_{i j k}^{\alpha \beta \gamma}=\sum_{\alpha+\beta+\gamma=\theta-1} M_{i j k}^{\alpha \beta \gamma}$.

2. The equations of $B_{i j k}^{\theta} \backslash\left\{M_{k j i}^{\theta-q_{i}-q_{j}-1 q_{i} q_{j}}\right\}$ are independent.

\footnotetext{
${ }^{2}$ Properties 1 are straightforward verifications; properties 2 can be shown to be arguments similar to those of Lemma 1.

${ }^{3}$ I.e., the block $B_{i j k}^{\theta}$ without the equation $N_{k i j}^{1 v u}$.
} 


\section{Appendix 2. The Spencer-Goldschmidt version of the CARTAN-KäHLER THEOREM (LINEAR CASE)}

Let $\pi_{k}: J_{k}(E) \longrightarrow J_{k-1}(E)$ be the canonical projection; since Ker $\pi_{k} \simeq S^{k} T^{*} \otimes$ $E$, one has the exact sequence,

$$
0 \longrightarrow S^{k} T^{*} \otimes E \stackrel{\varepsilon}{\longrightarrow} J_{k}(E) \stackrel{\pi_{k}}{\longrightarrow} J_{k-1}(E) \longrightarrow 0
$$

Let $F$ be another fiber bundle on $M$ and $P: \underline{E} \longrightarrow \underline{F}$ a $k^{t h}$-order linear differential operator. $P$ can be identified to a morphism of vector bundles $p_{o}(P): J_{k}(E) \longrightarrow F$. If the rank of $p_{o}(P)$ is constant and $R_{k}=\operatorname{Ker} p_{o}(P)$, one has the exact sequence

$$
0 \longrightarrow R_{k} \longrightarrow J_{k}(E) \stackrel{p_{o}(P)}{\longrightarrow} F \longrightarrow 0 .
$$

The l-th prolongation of $P$ at $x \in M$ is defined by

$$
\begin{aligned}
p_{l}(P): J_{k+l}(E) & \longrightarrow J_{l}(F), \\
j_{k+l}(s)(x) & \longmapsto j_{l}(P s)(x) .
\end{aligned}
$$

We set $R_{k+l}:=\operatorname{Ker} p_{l}(P) . R_{k+l}$ is called the space of formal solutions of order $k+l$.

Definition. The operator $P$ is formally integrable if the restrictions $\bar{\pi}_{k+l}: R_{k+l} \rightarrow$ $R_{k}$ are surjective for all $l \geq 1$.

In the analytic context formal integrability guarantees the existence of genuine local solutions "for any initial data". The following theorem is due to Ehrenpreis, Guillemin and Sternberg [4] and Malgrange [10]:

Theorem. Let $P$ be an analytic linear differential operator which is formally integrable; then $\forall x_{o} \in M$ and $\forall F_{o} \in R_{k, x_{o}}$, there exists a section $f$ of $E$ defined on a neighborhood $U$ of $x_{o}$, such that on $U$

$$
P(f)=0 \text { and }\left(j_{k} f\right)\left(x_{o}\right)=F_{o} .
$$

In order to prove the formal integrability of $P$, according to the definition we should verify an infinite number of conditions. The Cartan-Kähler theorem permits us to establish the formal integrability by a proof involving only a finite number of steps. In order to state this theorem, we introduce first the notion of involutivity (for simplicity we give here, as definition, a necessary and sufficient condition due to Serre).

The map $\sigma_{o}(P):=p_{o}(P) \circ \varepsilon:$

$$
\sigma_{o}(P): S^{k} T^{*} \otimes E \stackrel{\varepsilon}{\longrightarrow} J_{k}(E) \stackrel{p_{o}(P)}{\longrightarrow} F
$$

is called the symbol of $P$. The symbol of the $l$-th prolongation is defined by the composition

$$
\sigma_{l}(P): S^{k+1} T^{*} \otimes E \stackrel{\text { natural injection }}{\longrightarrow} S^{l} T^{*} \otimes S^{k} T^{*} \otimes E \stackrel{i d_{S^{l} T^{*}} \otimes \sigma_{o}}{\longrightarrow} S^{l} T^{*} \otimes F .
$$

In particular, $\sigma_{1}(P): S^{k+1} T^{*} \otimes E \longrightarrow T^{*} \otimes F$ is defined by

$$
i_{X} \sigma_{1}(P)(t)=\sigma_{o}(P)\left(i_{X} t\right) \forall X \in T M, \forall t \in S^{k+1} T^{*} \otimes E,
$$

where $i_{X}$ denotes the inner product by $X$.

Let $g_{l}:=\operatorname{Ker} \sigma_{l}$. For a basis $\left\{e_{1}, \ldots, e_{n}\right\}$ of $T_{x}$, and $j=1,2, \ldots, n-1$ we set

$$
\left(g_{o}\right)_{\left\{e_{1}, \ldots, e_{j}\right\}}:=\left\{A \in\left(g_{o}\right)_{x} \mid i_{e_{1}} A=0, \ldots, i_{e_{j}} A=0\right\} .
$$


Definition. A basis $\left\{e_{1}, \ldots, e_{n}\right\}$ of $T_{x}$ is called quasi-regular if

$$
\operatorname{dim}\left(g_{1}\right)_{x}=\operatorname{dim}\left(g_{o}\right)_{x}+\sum_{j=1}^{n-1} \operatorname{dim}\left(g_{o}\right)_{\left\{e_{1}, \ldots, e_{j}\right\}} .
$$

A differential operator is called involutive if at every point there exists a quasiregular basis.

One has:

Theorem. (Cartan-Kähler-Spencer-Goldschmidt). Let $P$ be a $k$-order linear differential operator. Suppose that the ranks of fiber bundles $R_{k}$ and $R_{k+1}$ are constant, and that the following conditions are satisfied.

1. $P$ is involutive.

2. $\bar{\pi}_{k+1}: R_{k+1} \longrightarrow R_{k}$ is surjective.

Then $P$ is formally integrable.

The involutivity insures that there are no more obstructions to extending formal solutions of order $k$ to formal solutions of higher orders.

In practice, the surjectivity of $\bar{\pi}_{k+1}$ is shown in the following way. One has the diagram of exact sequences

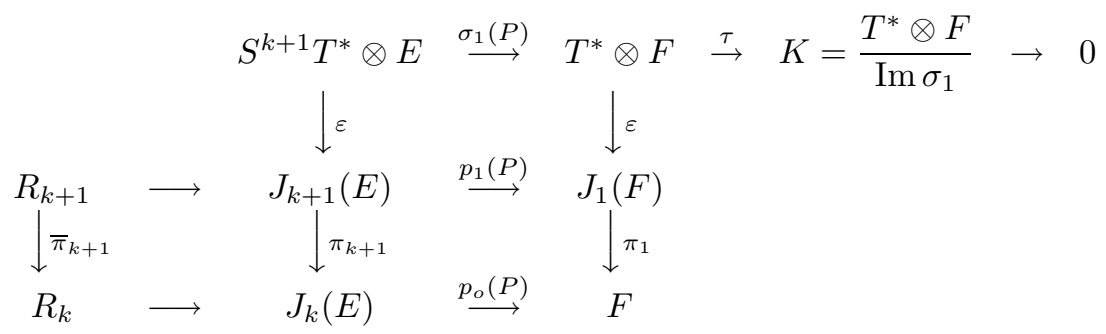

By a standard diagram-chasing argument, we obtain a map $\varphi: R_{k} \longrightarrow K$, and it is a straightforward verification that $\bar{\pi}_{k+1}: R_{k+1} \longrightarrow R_{k}$ is surjective if and only if $\varphi=0 . K$ is called the obstruction space.

In order to calculate $\varphi$, one makes use of an arbitrary connection $\nabla$ on the bundle $F, \nabla: \underline{F} \longrightarrow \underline{T^{*} \otimes F}$, that is, in jet notation,

$$
p_{o}(\nabla): J_{1}(F) \longrightarrow T^{*} \otimes F .
$$

With a slight abuse of notation one can write

$$
\varphi\left(j_{k}(s)(x)\right)=\tau(\nabla P(s))_{x}
$$

for $x \in M$ and every $s \in \underline{E}$ with $P(s)_{x}=0$.

To summarize, in order to prove the formal integrability, and consequently the existence of genuine local solutions in the analytic case, one needs to

I. prove involutivity,

II. construct the map $\tau$ and, for that, give a "good interpretation" of $K$, and

III. show that

$$
\tau(\nabla P(s))_{x}=0 \quad \forall s \in \underline{E} \text { for which } P(s)_{x}=0,
$$

where $\nabla$ is a connection on $F$. 


\section{REFERENCES}

[1] R. Bryant, S.S. Chern, R. Gardner, H. Goldschmidt, P. Griffiths, Exterior Differential Systems, Math. Sci. Res. Inst. Publ. 18, Springer-Verlag, New-York, Berlin, Heidelberg, 1991. MR 92h:58007

[2] P. Cabau, J. Grifone, M. Mehdi, Existence de lois de conservation dans le cas cyclique, Ann. Inst. Henri Poincaré Phys. Théor., 55 (1991), 789-803. MR 92k:58283

[3] E. Cartan, Les systèmes différentiels extérieurs et leurs applications géométriques, Paris: Hermann, 1945 MR 7:520d

[4] L. Ehrenpreis, V.W. Guillemin and S. Sternberg, On Spencer's estimate for $\delta$-Poincaré, Ann. of Math., 82 (1965), 128-138. MR 32:4709

[5] A. Frölicher, A. Nijenhuis, Theory of Vector-Valued Differential Forms, I, Nederl. Akad. Wetensch. Proc., 59 (1956) 338-359. MR 18:5569c

[6] J. Gasqui, Formal Integrability of Systems of Partial Differential Equations, Nonlinear Equations in Classical and Quantum Field Theory - N. Sanchez (ed.) Lect. Notes Physics, 226 (1985), Springer Verlag, 21-36. MR 86k:58140

[7] H. Goldschmidt, Integrability criteria for systems of non-linear partial differential equations Journ. Differ. Geom., 1 (1967) 269-307. MR 37:1746

[8] J. Grifone, M. Mehdi, Existence of Conservation Laws and Characterisation of Recursion Operators for Completely Integrable Systems. Prépublication n. 27 du Laboratoire de Topologie et Geometrie, Université de Toulouse III, Juin 1993

[9] P.D. Lax, Hyperbolic systems of conservation laws II, Comm. Pure Appl. Math. 10(1957), 537-566. MR 20:176

[10] B.Malgrange, Equations de Lie II, J. Diff. Geom., 7 (1972), 117-141. MR 48:5128

[11] F. Magri, C. Morosi, A geometrical characterization of integrable hamiltonian systems, Quaderno 5/19 Università di Milano (1984)

[12] M. Mehdi, Existence de lois de conservation et de systèmes bihamiltoniens Thèse, Toulouse (1991)

[13] H. Osborn, The Existence of Conservation Laws, I Ann. of Math., 69 (1959), 105-118. MR 21:760

[14] H. Osborn, Les lois de conservation, Ann. Inst. Fourier, Grenoble, 14 (1964), 71-82. MR 30: 2425

[15] D.C. Spencer, Overdetermined Systems of Linear Partial Differential Equations, Bull. A.M.S., 75 (1969), 179-239. MR 39:3533

[16] F.J. Turiel, Classification locale d'un couple de formes symplectiques Poisson-compatibles, C.R. Acad. Sci. Paris, Sér. I Math. 308 (1989), 573-578. MR 90g:58039

[17] F.J. Turiel, Classification locale des tenseurs de type $(1,1)$ presque plats, C.R. Acad. Sci. Paris, Sér. I Math. 319 (1994), 471-474. MR 95f:53061

Laboratoire Emile Picard, U.M.R. C.N.R.S. 5580, Département de Mathématiques, Université Paul Sabatier, 118, route de Narbonne, 31062 Toulouse Cedex, France

E-mail address: grifone@picard.ups-tlse.fr

Université Libanaise, Beyrouth, BP 13.5292 Chouran, Lebanon

E-mail address: lusc1@lara.cnrs.edu.lb 